\title{
Effect of Shear on Ultrasonic Flow Measurement Using Nonaxisymmetric Wave Modes
}

\author{
Yong Chen, ${ }^{1}$ Yiyong Huang, ${ }^{1}$ Xiaoqian Chen, ${ }^{1}$ and Dengpeng $\mathrm{Hu}^{2}$ \\ ${ }^{1}$ College of Aerospace Science and Engineering, National University of Defense Technology, Changsha 410073, China \\ ${ }^{2}$ Air Force Early Warning Academy, Wuhan 430019, China \\ Correspondence should be addressed to Xiaoqian Chen; chenxiaoqian@nudt.edu.cn
}

Received 12 March 2014; Revised 9 May 2014; Accepted 9 May 2014; Published 29 May 2014

Academic Editor: Valder Steffen Jr

Copyright (c) 2014 Yong Chen et al. This is an open access article distributed under the Creative Commons Attribution License, which permits unrestricted use, distribution, and reproduction in any medium, provided the original work is properly cited.

\begin{abstract}
Nonaxisymmetric wave propagation in an inviscid fluid with a pipeline shear flow is investigated. Mathematical equation is deduced from the conservations of mass and momentum, leading to a second-order differential equation in terms of the acoustic pressure. Meanwhile a general boundary condition is formulated to cover different types of wall configurations. A semianalytical method based on the Fourier-Bessel theory is provided to transform the differential equation to algebraic equations. Numerical analysis of phase velocity and wave attenuation in water is addressed in the laminar and turbulent flow. Meanwhile comparison among different kinds of boundary condition is given. In the end, the measurement performance of an ultrasonic flow meter is demonstrated.
\end{abstract}

\section{Introduction}

Wave propagation in an inviscid fluid with a shear mean flow has been studied mostly by numerical and semianalytical methods $[1,2]$ which is of considerable interest in industrial applications such as noise damping [3,4] and ultrasonic flow meter $[5,6]$, to mention a few. On the neglect of the fluid viscosity and thermal conductivity, Pridmore-Brown [7] firstly established the governing equation of the transverse modes in a shear mean flow confined by a rectangular duct. Mungur and Plumblee [8] derived the corresponding convected wave equation in the case of a circular pipeline.

Following the contribution of Mungur and Plumblee [8], various aspects of wave propagation in the circular pipeline containing the shear flow were studied by many researchers, such as Gogate and Munjal [9], Ko [10], Eversman [11], Agarwal and Bull [12], and Pagneux and Froelich [13]. Boucheron et al. [2] made a comprehensive review of axisymmetric acoustic wave propagating in the rigid-walled pipeline. In the case of a lined-walled pipeline where the effect of acoustic impedance of the wall was considered, theoretical and numerical contributions were made by Rienstra and Darau [3], Rienstra [14], Smith et al. [15], Brambley et al. [16], and Buske et al. [17]. Some experiment data can be found in the work of Marx et al. [18].

In the application of an ultrasonic flow measurement, particular considerations are given to improve the measurement performance. Due to the complexity of wave propagation in the pipeline flow, various compensation methods in the engineering aspect have been provided, which was comprehensively summarized by Chen et al. [5]. On the other hand, theoretical analysis based on the wave equation was conducted by Lechner [19], Willatzen [20-24], and Chen et al. [6]. Based on the axisymmetric acoustic wave, the performance for the low Mach flow measurement was analyzed in the abovementioned paper. Although the effect of the acoustic impedance at the wall on the measurement performance was analyzed by Willatzen [23], the features of wave absorption at the wall were not addressed.

The present paper concentrates on the nonaxisymmetric acoustic wave propagating in the inviscid fluid with the presence of a shear mean flow confined by a circular pipeline. Particular attention is given to the influence of the acoustic impedance on the wave propagation and measurement performance. Mathematical formulation starts from the conservations of mass and momentum which leads to a 


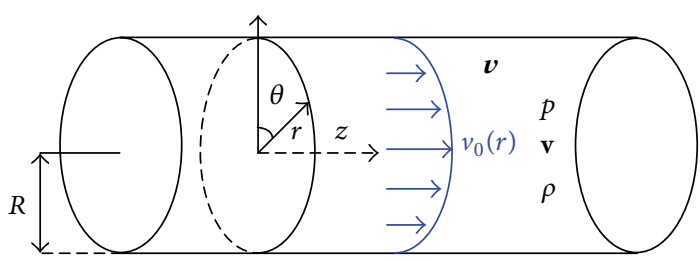

FIGURE 1: (Color online) Configuration of the cylindrical coordinate system (the flow profile is assumed to be sheared).

second-order differential equation in terms of the acoustic pressure. The boundary condition is then discussed under the assumptions of rigid wall, free-boundary wall (pressurerelease wall) $[25,26]$, and lined wall. In the previous research, the authors introduced a general solution based on the Fourier-Bessel theory $[27,28]$ to study the axisymmetric acoustic wave propagating along the rigid-walled pipeline $[29]$ and heating pipeline $[6,30]$. Such method was extended to handle the nonaxisymmetric wave propagation. Numerical analysis of phase velocity and wave attenuation is addressed in the laminar and turbulent flow. Furthermore, the measurement performance of an ultrasonic flow meter based on the wave propagation is demonstrated.

\section{Mathematical Formulation}

In this section, the convected equation of the nonaxisymmetric acoustic wave propagating in the inviscid fluid flowing through a circular pipeline is mathematically deduced as shown in Figure 1. The flow profile is assumed to be shear and denoted by $v_{0}(r)$. The radial, circumferential, and axial coordinates are expressed by $r, \theta$, and $z$, respectively. The fluid density, velocity, and pressure are denoted by $\rho, \mathbf{v}$, and $p$, respectively. $R$ represents the pipeline inner radius. In what follows, the thermoviscous effect is not taken into account, and then the entropy perturbation during wave propagation can be taken to be zero.

2.1. Governing Equation. In the presence of wave propagation, acoustic disturbances impose small fluctuating terms on the fluid's ambient variables which can be expressed by $\rho^{\prime}$, $\mathbf{v}^{\prime}\left(=\left[v_{r}^{\prime}, v_{\theta}^{\prime}, v_{z}^{\prime}\right]\right)$, and $p^{\prime}$. If these fluctuations are assumed to be linear, according to the work of Mungur and Plumblee [8] and Chen et al. [31], the governing equation of the acoustic wave can be expressed by

$$
\begin{aligned}
\nabla^{2} p^{\prime} & -\frac{1}{c_{0}^{2}} \frac{\partial^{2} p^{\prime}}{\partial t^{2}}-2 \frac{M}{c_{0}} \frac{\partial^{2} p^{\prime}}{\partial t \partial z} \\
& -M^{2} \frac{\partial^{2} p^{\prime}}{\partial z^{2}}+2 \rho_{0} c_{0} \frac{\mathrm{d} M}{R \mathrm{~d} x} \frac{\partial v_{r}^{\prime}}{\partial z}=0,
\end{aligned}
$$

where $c_{0}$ and $M(x)=v_{0}(x) / c_{0}$ represent the adiabatic sound speed and the local Mach number, respectively. $x$ is the normalized radial coordinate through $x=r / R$.

The fluctuating quantities can be taken in the form of $\exp \left[i\left(\omega t-k_{0} K z+m \theta\right)\right]$, where $\omega(=2 \pi f)$ is the angular frequency, $K$ the dimensionless axial wavenumber, $k_{0}=\omega / c_{0}$ the free-space wavenumber, and $m$ is the circumferential mode which is constrained to be integral due to the periodic wave propagation. The radial component of the acoustic velocity may be expressed by

$$
v_{r}^{\prime}=\frac{i}{\rho_{0} \omega R(1-K M)} \frac{\partial p^{\prime}}{\partial x} .
$$

Through expressing the acoustic pressure as $\varphi_{p}(x) \exp [i(\omega t-$ $\left.k_{0} K z+m \theta\right)$ ], where $\varphi_{p}(x)$ is the radial component of the acoustic pressure, (1) can be simplified to

$$
\begin{gathered}
(1-K M)\left(\frac{\mathrm{d}^{2} \varphi_{p}}{\mathrm{~d} x^{2}}+\frac{1}{x} \frac{\mathrm{d} \varphi_{p}}{\mathrm{~d} x}-\frac{m^{2}}{x^{2}} \varphi_{p}\right)+2 K \frac{\mathrm{d} M}{\mathrm{~d} x} \frac{\mathrm{d} \varphi_{p}}{\mathrm{~d} x} \\
+k_{0}^{2} R^{2}(1-K M)\left[(1-K M)^{2}-K^{2}\right] \varphi_{p}=0,
\end{gathered}
$$

while the radial acoustic velocity is

$$
v_{r}^{\prime}=\frac{i}{\rho_{0} \omega R(1-K M)} \frac{\mathrm{d} \varphi_{p}}{\mathrm{~d} x} \exp \left[i\left(\omega t-k_{0} K z+m \theta\right)\right] .
$$

Obviously, the nonaxisymmetric acoustic wave propagating in the inviscid fluid with the existence of the pipeline shear flow can be governed by a second-order differential equation with respect to the acoustic pressure. To solve the deduced equation, the boundary condition should be discussed.

2.2. Boundary Condition. On the ignorance of the fluid viscosity and thermal conductivity, the boundary condition can be expressed by the standard Ingard-Myers condition $[3,16]$ :

$$
\frac{\partial v_{r}}{\partial t}=\left(\frac{\partial}{\partial t}+c_{0} M \frac{\partial}{\partial z}\right) \frac{P^{\prime}}{Z}
$$

where $Z$ denotes the acoustic impedance at the wall and is assumed constant in this paper. Due to the vanishing of the shear flow velocity at the wall, one obtains $v_{r}=p^{\prime} / Z$. Then substituting (4) into the boundary condition yields

$$
\frac{\mathrm{d} \varphi_{p}}{\mathrm{~d} x}+i \frac{\rho_{0} \omega R}{Z} \varphi_{p}=0 .
$$

According to the work of Rienstra and Darau [3], Brambley et al. [16], and Marx et al. [18], the constant acoustic impedance can be modeled as an enhanced Helmholtz resonator

$$
Z(\omega)=Z_{0}+i \omega \widetilde{m}-i \rho_{0} c_{0} \cot \left(\frac{\omega D}{c_{0}}\right),
$$

where $D$ is the lining depth, $Z_{0}$ is the damping resistance, and $\widetilde{m}$ is the inertance. In the case of a rigid wall $(Z(\omega)=\infty),(6)$ is simplified to

$$
\frac{\mathrm{d} \varphi_{p}(x)}{\mathrm{d} x}=0, \quad \text { at } x=1 .
$$


In the situation of a free-boundary wall $(Z(\omega)=0),(6)$ is transformed to

$$
\varphi_{p}(x)=0, \quad \text { at } x=1 .
$$

As a result, the boundary condition can be generally expressed by

$$
\frac{\mathrm{d} \varphi_{p}(x)}{\mathrm{d} x}+\gamma \varphi_{p}(x)=0, \quad \text { at } x=1,
$$

where $\gamma$ is independent of the radial coordinate. Specifically $\gamma=0, \gamma=\infty$, and $0<|\gamma|<\infty$ represent the rigid wall, free-boundary wall, and lined wall, respectively.

\section{Solution Based on Fourier-Bessel Theory}

In this section, a solution based on the Fourier-Bessel theory, which is mathematically complete and orthogonal in the Lebesgue space $\mathscr{L}_{x}^{2}(0,1)[27,28]$, is proposed to solve (3) with the boundary condition (10).

Mathematically, the bounded continuous function $\varphi_{p}(x)$ in the domain $x \in[0,1]$ may be expressed in terms of a Fourier-Bessel sequence

$$
\varphi_{p}(x)=\sum_{j=1}^{\infty} C_{j} J_{m}\left(\lambda_{j}^{p} x\right),
$$

where $J_{m}\left(\lambda_{j}^{p} x\right)$ denotes the $m$ th-order Bessel function of the first kind and $m$ corresponds to the circumferential mode. Substitution into (10) leads to

$$
\frac{\lambda_{j}^{p}}{2}\left(J_{m-1}\left(\lambda_{j}^{p}\right)-J_{m+1}\left(\lambda_{j}^{p}\right)\right)+\gamma J_{m}\left(\lambda_{j}^{p}\right)=0,
$$

which can determine the coefficients $\lambda_{j}^{p}$. Meanwhile, the orthogonality $[27,28]$ of the Fourier-Bessel sequence may be represented by

$$
\begin{gathered}
\int_{0}^{1} J_{m}\left(\lambda_{i}^{p} x\right) J_{m}\left(\lambda_{j}^{p} x\right) x \mathrm{~d} x \\
=\left\{\frac{1}{8}\left[\left(J_{m-1}\left(\lambda_{j}^{p}\right)-J_{m+1}\left(\lambda_{j}^{p}\right)\right)\right]^{2}\right. \\
\left.+\frac{\left(\left(\lambda_{j}^{p}\right)^{2}-m^{2}\right)}{2\left(\lambda_{j}^{p}\right)^{2}} J_{m}^{2}\left(\lambda_{j}^{p}\right)\right\} \delta_{i j},
\end{gathered}
$$

where $\delta_{i j}$ is the Kronecker delta function. If the acoustic pressure $\varphi_{p}(x)$ is given, the corresponding coefficients $C_{j}$ can be obtained by

$$
\begin{aligned}
C_{j}= & 8\left(\lambda_{j}^{p}\right)^{2} \times\left(\left(\lambda_{j}^{p}\right)^{2}\left(J_{m-1}\left(\lambda_{j}^{p}\right)-J_{m+1}\left(\lambda_{j}^{p}\right)\right)^{2}\right. \\
& \left.+4\left(\left(\lambda_{j}^{p}\right)^{2}-m^{2}\right) J_{m}^{2}\left(\lambda_{j}^{p}\right)\right)^{-1} \\
& \times \int_{0}^{1} \varphi_{p}(x) J_{m}\left(\lambda_{j}^{p} x\right) x \mathrm{~d} x
\end{aligned}
$$

which shows that $C_{j}$ is independent of the radial coordinate.
Obviously, the Bessel functions $J_{m}\left(\lambda_{j}^{p} x\right)$ are solutions of cylindrical waves without flow, which are not the eigenfunctions with shear flow. While the eigenfunctions without flow $\left(J_{m}\left(\lambda_{j}^{p} x\right)\right)$ are orthogonal and complete, the eigenfunctions with shear flow may be not. As a result, (11) is a valid orthogonal representation but is not an eigenfunction decomposition. It specifies pseudo-modes that are the approximate representation of the acoustic waves with shear in terms of acoustic waves without shear. The calculation of the coefficients $C_{j}$ and pseudo-eigenvalues $\lambda_{j}^{p}$ minimizes the mean square error of the representation. As a result, substituting (11) into (3) yields

$$
\begin{aligned}
\sum_{j=1}^{\infty}\left(R^{2} k_{0}^{2} K^{2}-R^{2} k_{0}^{2}+\left(\lambda_{j}^{p}\right)^{2}\right) J_{m}\left(\lambda_{j}^{p} x\right) C_{j} \\
=\sum_{j=1}^{\infty}\left\{\left[\left(R^{2} k_{0}^{2} K^{2}+\left(\lambda_{j}^{p}\right)^{2}-3 R^{2} k_{0}^{2}\right) K M\right.\right. \\
\left.+3 R^{2} k_{0}^{2} K^{2} M^{2}-R^{2} k_{0}^{2} K^{3} M^{3}\right] J_{m}\left(\lambda_{j}^{p} x\right) \\
\left.+\lambda_{j}^{p} K \frac{\mathrm{d} M}{\mathrm{~d} r}\left(J_{m-1}\left(\lambda_{j}^{p} x\right)-J_{m+1}\left(\lambda_{j}^{p} x\right)\right)\right\} C_{j} .
\end{aligned}
$$

In the above equation, the properties of Bessel function are used $[27,28]$. Similar deductions can be found by the authors' previous papers $[6,29,30]$. Multiplying $J_{m}\left(\lambda_{i}^{p} x\right) x \mathrm{~d} x$ and then integrating in the domain $x \in[0,1]$ result in

$$
\begin{aligned}
&\left(R^{2} k_{0}^{2} K^{2}-\right.\left.R^{2} k_{0}^{2}+\left(\lambda_{i}^{p}\right)^{2}\right) C_{i} \\
&+\sum_{j=1}^{\infty}\left[\left(R^{2} k_{0}^{2} K^{3}+\left(\lambda_{j}^{p}\right)^{2} K-3 R^{2} k_{0}^{2} K\right)(H A)_{i}^{j}\right. \\
&+3 R^{2} k_{0}^{2} K^{2}(H B)_{i}^{j} \\
&\left.+R^{2} k_{0}^{2} K^{3}(H C)_{i}^{j}+\lambda_{j}^{p} K(H D)_{i}^{j}\right] C_{j}=0,
\end{aligned}
$$

where

$$
\begin{aligned}
& (H A)_{i}^{j}=F_{i} \int_{0}^{1} M J_{m}\left(\lambda_{j}^{p} x\right) J_{m}\left(\lambda_{i}^{p} x\right) x \mathrm{~d} x, \\
& (H B)_{i}^{j}=F_{i} \int_{0}^{1} M^{2} J_{m}\left(\lambda_{j}^{p} x\right) J_{m}\left(\lambda_{i}^{p} x\right) x \mathrm{~d} x, \\
& (H C)_{i}^{j}=F_{i} \int_{0}^{1}-M^{3} J_{m}\left(\lambda_{j}^{p} x\right) J_{m}\left(\lambda_{i}^{p} x\right) x \mathrm{~d} x, \\
& (H D)_{i}^{j}=F_{i} \times \int_{0}^{1} \frac{\mathrm{d} M}{\mathrm{~d} x}\left(J_{m-1}\left(\lambda_{j}^{p} x\right)-J_{m+1}\left(\lambda_{j}^{p} x\right)\right) \\
& \quad \times J_{m}\left(\lambda_{i}^{p} x\right) x \mathrm{~d} x, \\
& F_{i}=-8\left(\lambda_{i}^{p}\right)^{2} \times\left(\left(\lambda_{i}^{p}\right)^{2}\left(J_{m-1}\left(\lambda_{i}^{p}\right)-J_{m+1}\left(\lambda_{i}^{p}\right)\right)^{2}\right. \\
& \left.+4\left(\left(\lambda_{i}^{p}\right)^{2}-m^{2}\right) J_{m}^{2}\left(\lambda_{i}^{p}\right)\right)^{-1} .
\end{aligned}
$$


Obviously, given specific local Mach profile $M(x)$, acoustic angular frequency $\omega$, pipeline radius $R$, fluid density $\rho_{0}$, and the orthogonal Bessel sequence $J_{m}\left(\lambda_{j}^{p} x\right)$ with the corresponding eigenvalues $\lambda_{j}^{p}, n=1,2, \ldots, N$ being the roots of (12), expanding (16) gives a set of homogeneous algebraic equations with the unknown parameters $C_{j}$ and the dimensionless axial wavenumber $K$. It is obvious that if the number of equations expanded from (16) is $N$, the number of coefficients is $N+1$. Clearly, the problem is homogeneous and the wave amplitude can be multiplied by an arbitrary constant.

While the specific coefficients $C_{j}$ cannot be solved from (16), the dimensionless axial wavenumber $K$ can be obtained. Furthermore, the ratio of all $C_{j}$ to one of them such as $C_{j} / C_{1}$ (if $C_{1} \neq 0$ ) can be calculated. As we are interested in the wavenumber $K$, by taking the coefficients $C_{j}$ as unknown variables with $\mathbf{X}=\left[C_{1}, \ldots, C_{N}\right]^{\mathrm{T}}$ (the subscript " $\mathrm{T}$ " means the operation of matrix transpose), (16) can be written as

$$
\mathbf{G}(K) \mathbf{X}=\mathbf{0}
$$

where $\mathbf{G}(K)$ is a $N \times N$ matrix whose element is a function of the dimensionless axial wavenumber. From (14), it can be learned that the coefficients do not vanish simultaneously, leading to the condition of $\mathbf{X} \neq \mathbf{0}$. The existence condition of a nontrivial solution to (16) is then the vanishment of the corresponding determinant

$$
\operatorname{det}(\mathbf{G}(K))=0
$$

which can be solved by various numerical methods. A Newton-related method can be found in the authors' papers $[6,29,30]$.

\section{Numerical Analysis of Wave Propagation}

In this section, wave propagation in water is numerically studied. The corresponding constant parameters are $\rho_{0}=$ $1000 \mathrm{~kg} / \mathrm{m}^{3}, c_{0}=1500 \mathrm{~m} / \mathrm{s}, f=1 \mathrm{MHz}$, and $R=4 \mathrm{~mm}$. Following the work of Rienstra and Darau [3], the parameters of acoustic impedance are assumed to be $Z_{0}=2 \rho_{0} c_{0}, D=$ $2 \mathrm{~mm}$, and $\widetilde{m}=0.02 \rho_{0}+(1 / 3) \rho_{0} D$. The laminar flow profile can be described by a Poisson's equation

$$
M(x)=2 \bar{M}\left(1-x^{2}\right)
$$

while the turbulent flow profile can be represented as the seventh-root law

$$
M(x)=\frac{60}{49} \bar{M}(1-x)^{1 / 7}
$$

where $\bar{M}$ is the cross-section average Mach number. Due to the acoustic impedance of the lined wall, the acoustic energy is absorbed at the wall. The following analysis focuses on the phase velocity $\left(c_{p}\right)$ and attenuation coefficient $(A)$

$$
c_{p}=\frac{c_{0}}{K_{\mathrm{R}}}, \quad A=\left|8.686 k_{0} K_{\mathrm{I}}\right|,
$$

where the subscripts " $R$ " and "I" denote the real and imaginary components of the axial wavenumber, respectively. To get a dimensionless description of the phase velocity, the relative phase velocity $c_{p} / c_{0}=1 / K_{\mathrm{R}}$ is used in the following discussion. Furthermore, the number of Bessel functions is set to $N=30$ and a convincible calculation convergence of the axial wavenumber $K$ can be reached $[6,29,30]$. In what follows, the mean Mach number is assumed to be in the interval $0<\bar{M}<0.2$. Readers may calculate the axial wavenumber for any value of the mean Mach number using presented method.

\subsection{Phase Velocity}

4.1.1. Comparison among Different Kinds of Boundary Condition. Figure 2 represents the relative phase velocity under the three different wall configurations (explained in Section 2.2) in the laminar flow, while Figure 3 shows the corresponding relative phase velocity in the turbulent flow. Numerical comparison is imposed on the relative phase velocity of the first four axisymmetric $(m=0)$ radial pseudomodes as a function of the average Mach number. In the legends, "down/up" means wave propagation in the downstream/upstream direction while "free/gen/rigid" represents the free-boundary/lined/rigid wall configuration. The first four axisymmetric radial pseudo-modes are denoted by "1," “2," “3," and " 4 ," respectively.

When the fluid is at rest, the relative phase velocity of each axisymmetric acoustic mode in the rigid wall turns out to be the largest among the three types of the boundary condition. The relative phase velocity in the lined-walled pipeline shows the smallest. In the case of the high modes, the difference of the relative phase velocity among the three boundary types becomes more distinct. Physically speaking, the complicated refraction and reflection at the wall lead to the existence of the high modes ("2," “ 3 ," and “4”). With a higher mode, the corresponding wave confronts more complex reflections and refractions. The wall has influence on the acoustic wave and then becomes more obvious, leading to a slower propagation speed in the lined wall. However, the wall influence on the fundamental mode (" 1 ") is slight.

In the case of a moving fluid, the relative phase velocity increases with the average Mach number in the downstream propagation but decreases in the upstream propagation. In the turbulent flow, the relative phase velocity in the downstream propagation is semisymmetric to that in the upstream propagation with respect to the case of the static fluid. However, the symmetric relationship is altered in the laminar flow due to the shear effect. Such phenomenon is more obvious in the fundamental mode wave than the other higher acoustic modes.

Physically speaking, with the increase of the average flow Mach number, the convective flow (uniform flow) accelerates the downstream propagation as the propagation direction is along the flow direction but decelerates the upstream propagation. The consequence is that the relative phase velocity in the downstream and upstream propagation due to the flow convection shows to be symmetric with respect 


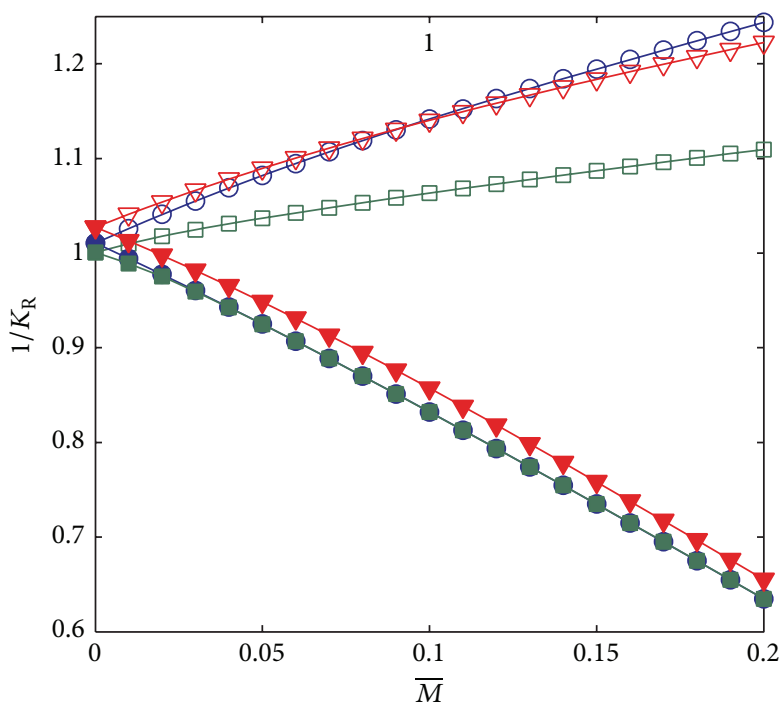

(a)

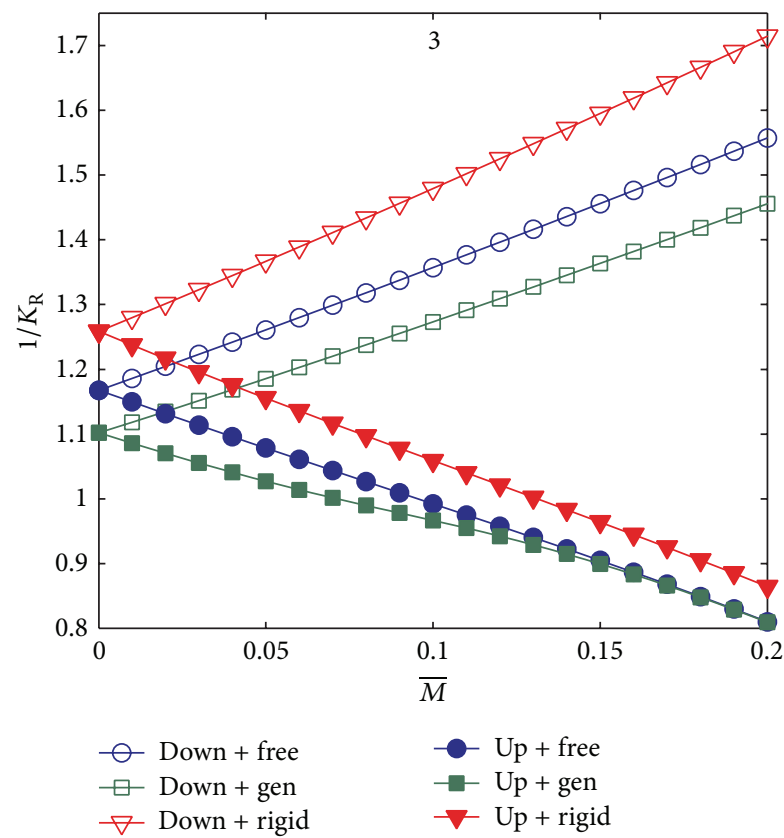

(c)

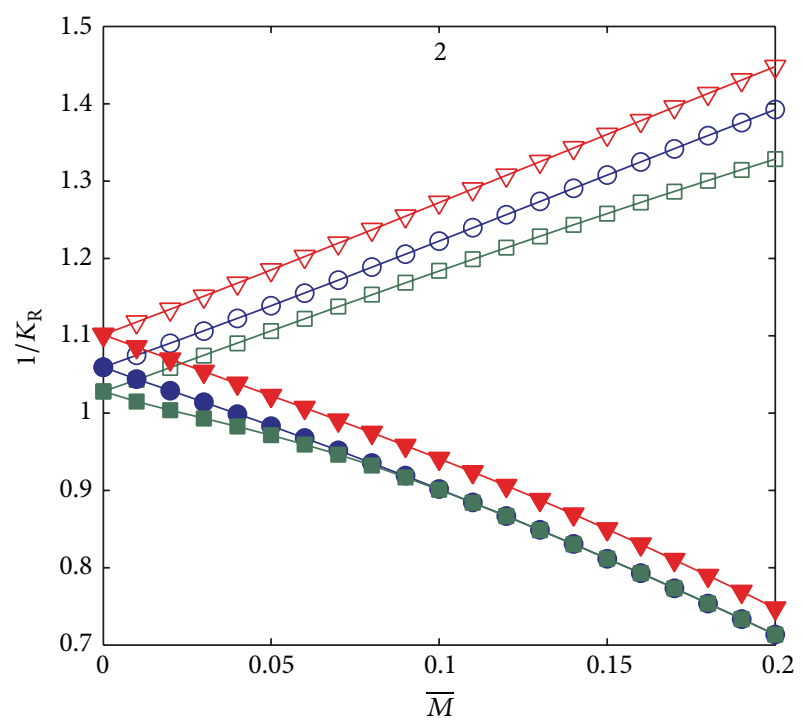

(b)

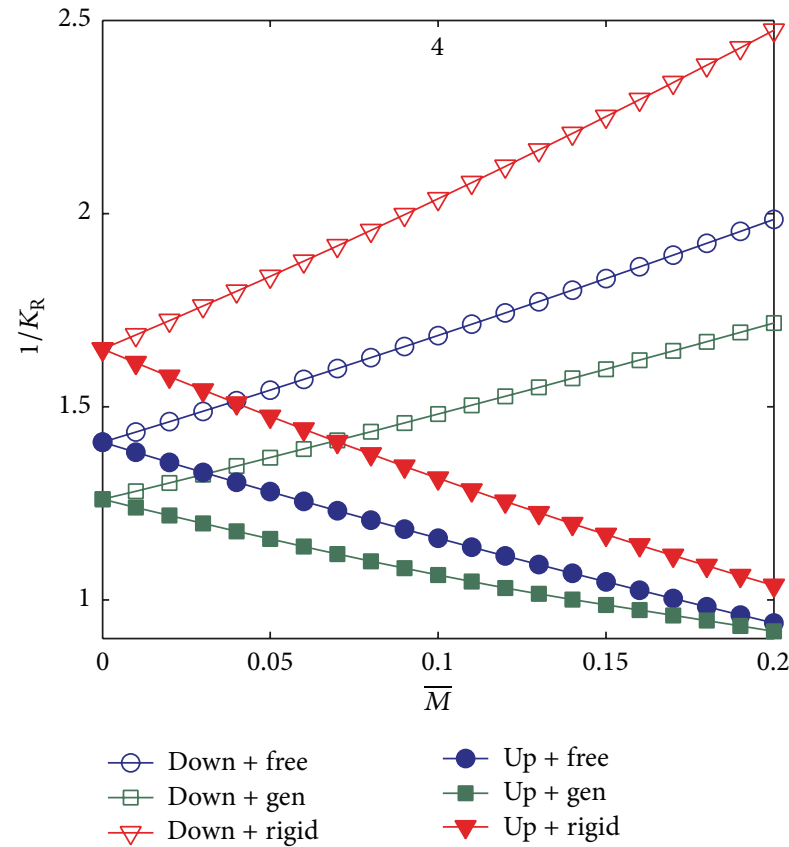

(d)

FIGURE 2: (Color online) Comparison of the relative phase velocity of the first four axisymmetric pseudo-modes with three different types of boundary condition in the laminar flow.

to the static fluid case. Such symmetric relationship can be found in Figure 3 as the turbulent flow profile is similar to the convective flow profile.

In the laminar flow, the phenomenon becomes more complicated as the shear effect alters the abovementioned symmetric relationship. From Figure 2(a), it can be inferred that the shear effect channels the fundamental mode ("1") into a narrow layer near the wall in the downstream propagation. In the upstream propagation, the acoustic wave is channeled into a narrow layer near the pipeline center [7]. As the local Mach number near the wall is smaller than that near the pipeline center, the contribution of the flow profile on the propagation speed is slight in the downstream propagation. In the upstream propagation, the increased local Mach number near the center heavily decelerates the propagation speed. For the high acoustic modes ("2," "3," and "4"), the shear effect channels the acoustic wave into a narrow layer next to the centerline. As a result, the local Mach number near the pipeline center accelerates the downstream propagation but decelerates the upstream propagation. Such phenomenon can be seen by comparing the relative phase velocity between Figures 2 and 3 . It should be noticed that the shear effect 


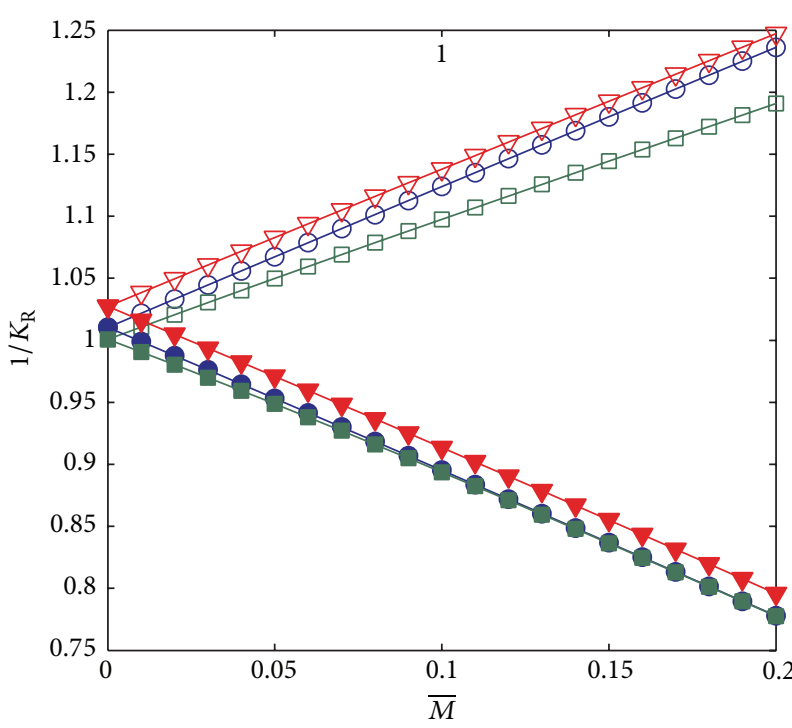

(a)

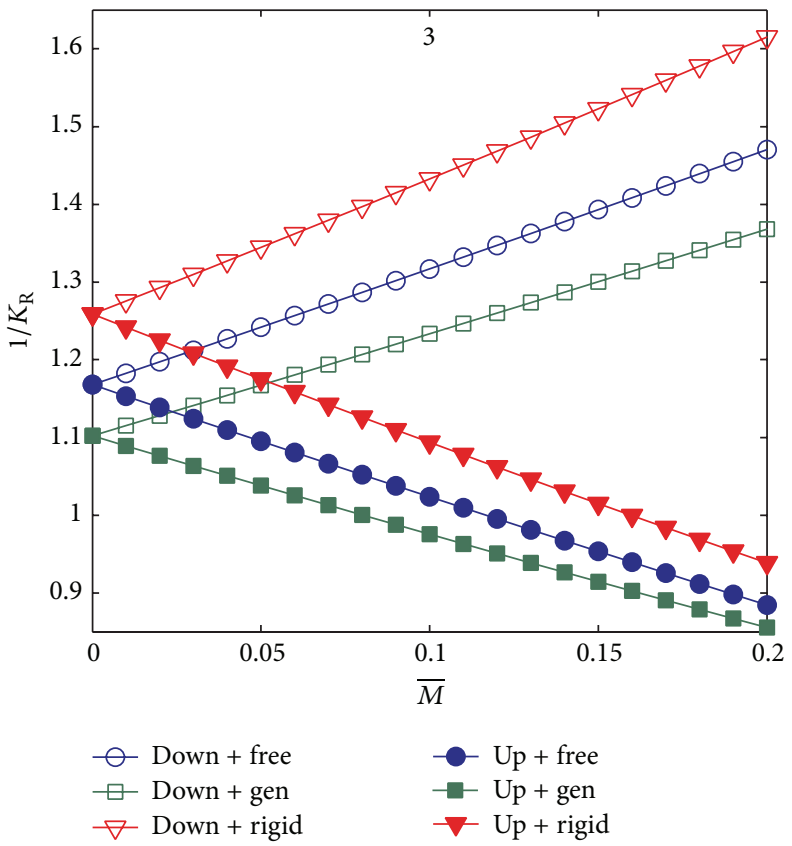

(c)

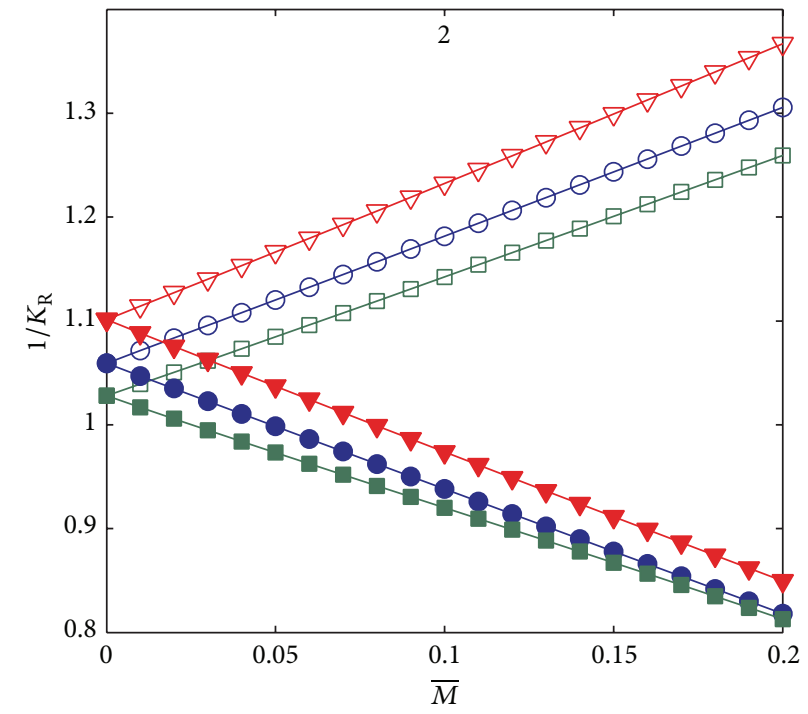

(b)

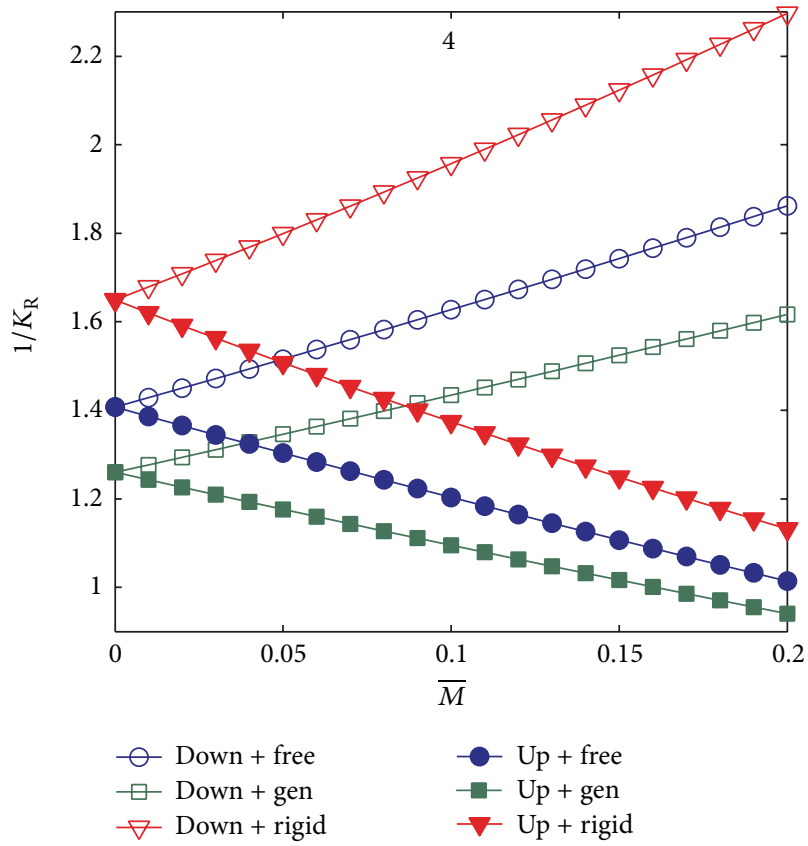

(d)

Figure 3: (Color online) Comparison of the relative phase velocity of the first four axisymmetric pseudo-modes with three different types of boundary condition in the turbulent flow. See Figure 2 for legend's interpretation.

exists in both the laminar and turbulent flow. However, such influence is more significant in the laminar flow as the fluid vorticity is larger for the parabolic velocity profile (20) than for the logarithmic (21) flow profile.

It should be noticed that the shear effect on wave propagation confined by the free-boundary wall is less obvious. On the other hand, the existence of acoustic impedance at the wall prefers to regulate the wave propagation to a narrow layer near the pipeline wall. As a result, in the downstream propagation, the local Mach number near the pipeline wall slightly accelerates the propagation speed compared with that near the pipeline center. In the upstream propagation, the local flow profile near the wall weakens the decreasing rate of the relative phase velocity due to the flow convection. Such phenomenon can be found in both the laminar and turbulent flow as shown in Figures 2 and 3.

4.1.2. Comparison among Different Circumferential Modes. Figure 4 compares the relative phase velocity of the first four axial pseudo-modes ("1," "2," "3," and "4") among the three different circumferential modes $(m=0, m=1$, and $m=2)$ 


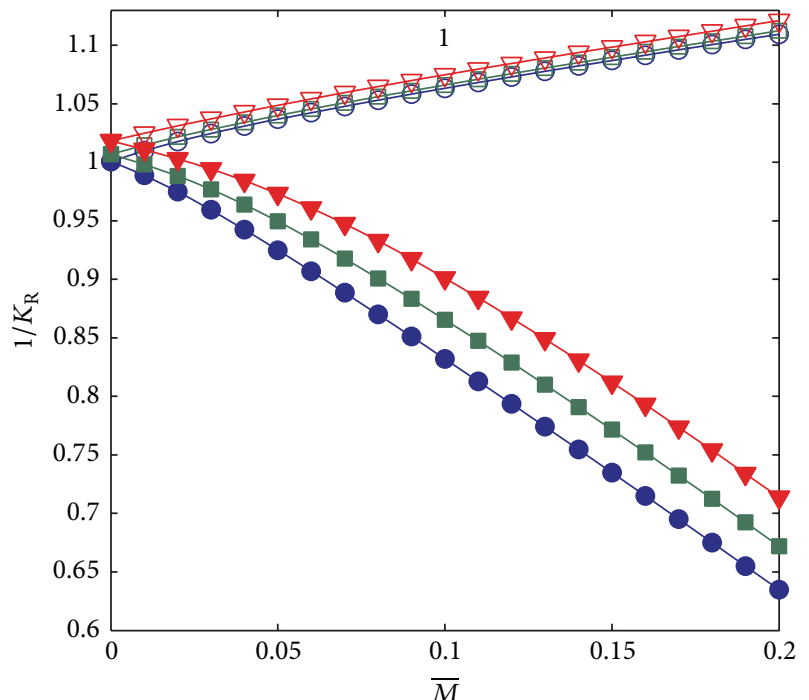

(a)

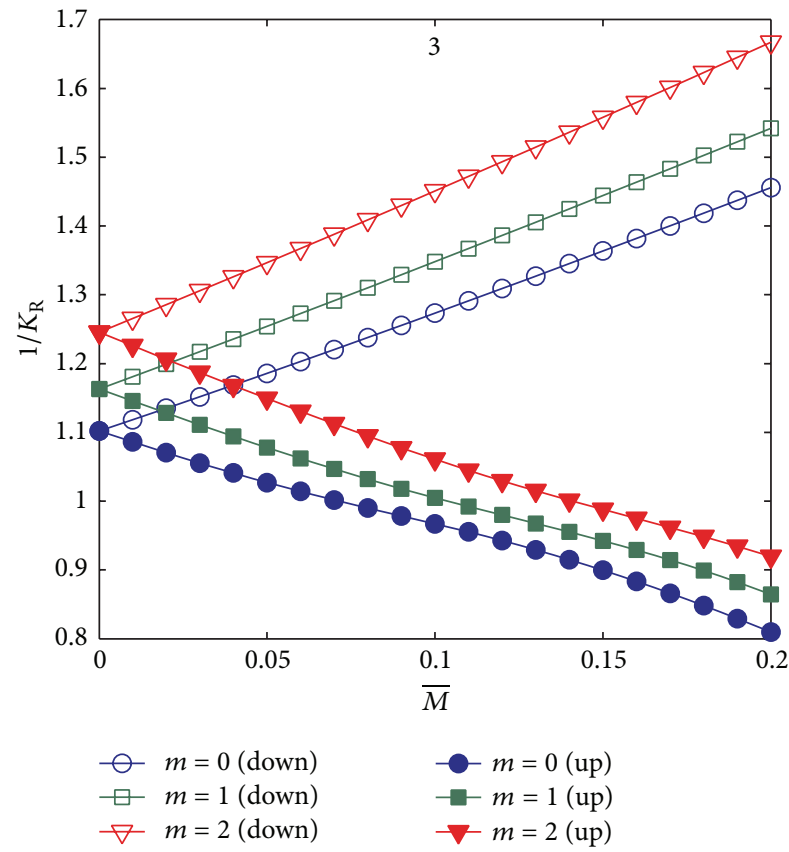

(c)

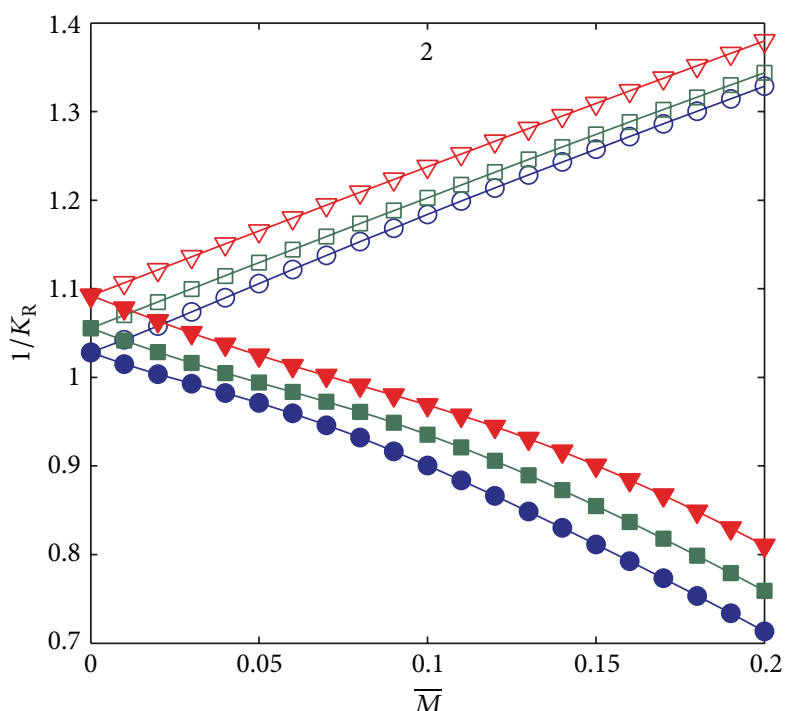

(b)

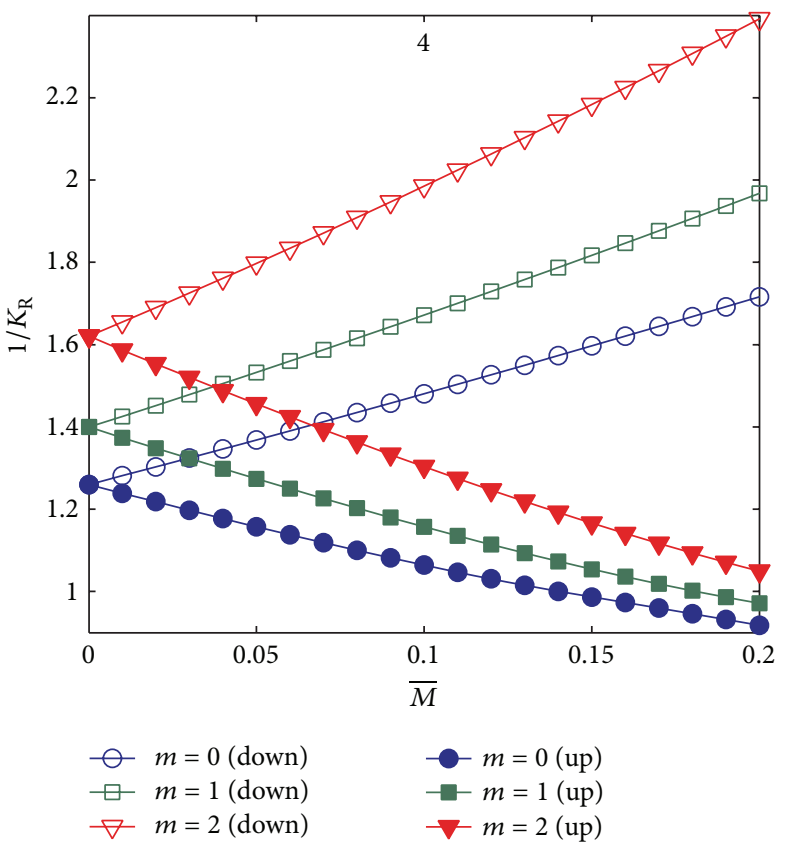

(d)

FIGURE 4: (Color online) Comparison of the relative phase velocity of the first four axial pseudo-modes among the three circumferential modes $(m=0, m=1$, and $m=2)$ in the laminar flow confined by the lined-walled pipeline.

in the laminar flow confined by the lined-walled pipeline, while Figure 5 shows the corresponding comparisons in the turbulent flow.

In the static fluid $(\bar{M}=0)$, the relative phase velocity of a higher circumferential mode is larger. In the case of a higher axial mode, the difference of the relative phase velocity among the three circumferential modes becomes more visible. In the presence of a flow, the flow convection speeds up the phase velocity of the acoustic wave propagating in the downstream direction but slows down the relative phase velocity in the upstream propagation. The influence of flow convection on each acoustic mode can be explicitly found in the case of turbulent flow in Figure 5 as the core profile of the turbulent flow is nearly the same with the uniform flow profile.

In the laminar flow (Figure 4), the tendency becomes more complicated compared with that in the turbulent flow as shown in Figure 5. For the first axial pseudo-mode (Figure 4(a)), the shear effect regulates each circumferential mode into a narrow layer next to the wall in the downstream propagation; meanwhile, it channels each circumferential mode into a narrow layer next to the pipeline center in the upstream propagation. By comparing the tendencies of 


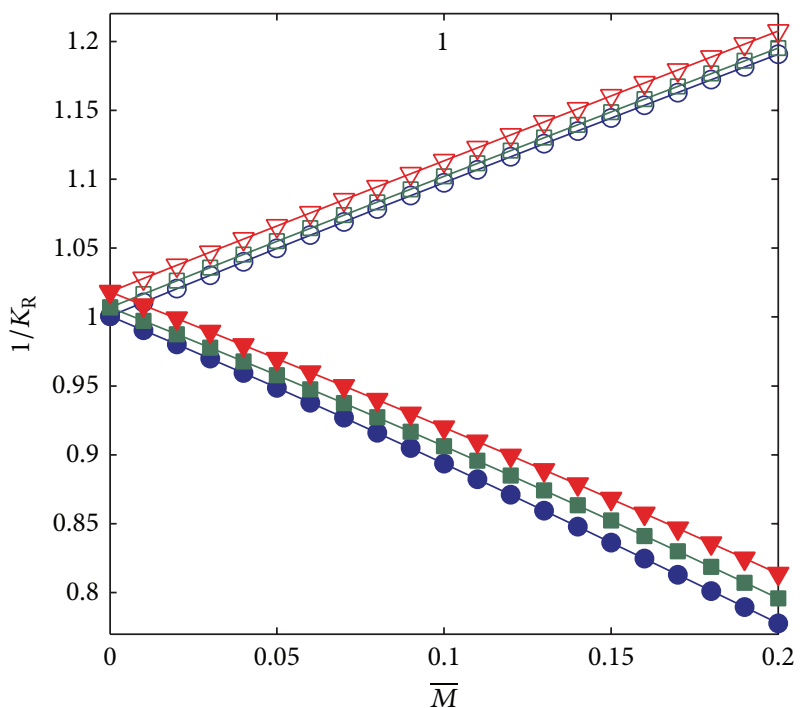

(a)

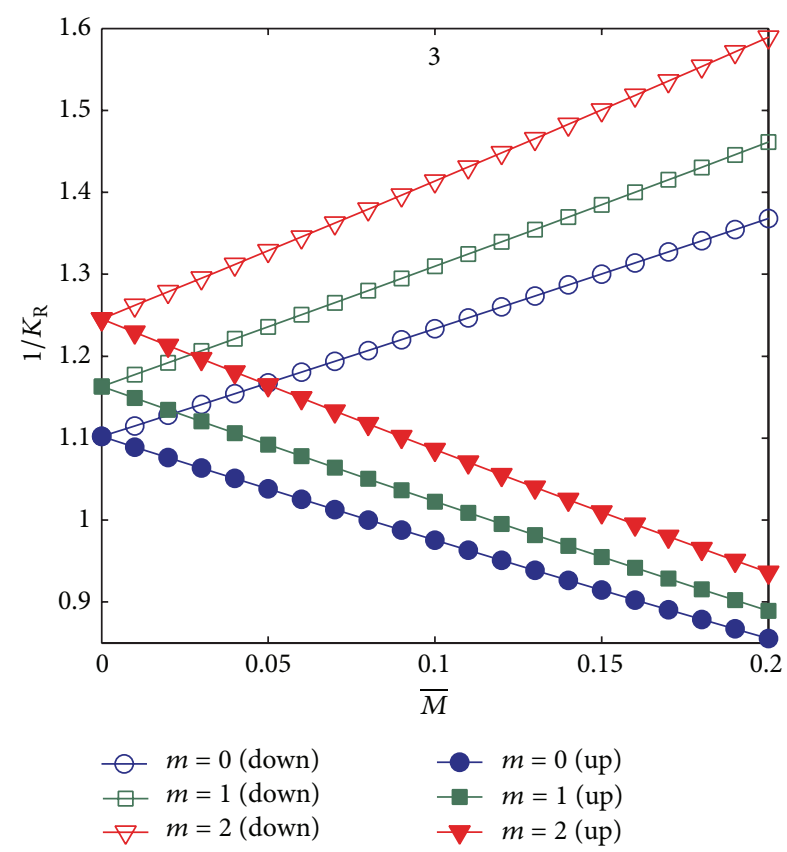

(c)

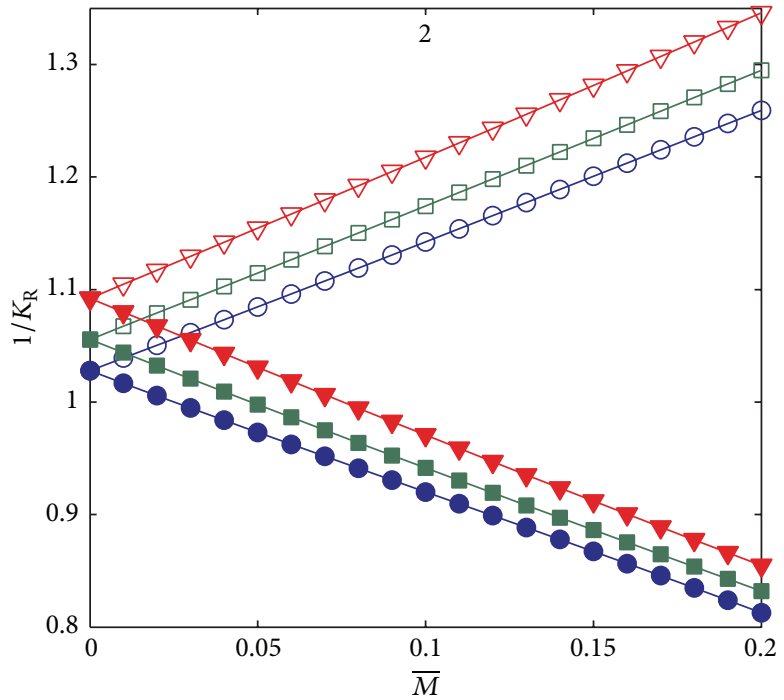

(b)

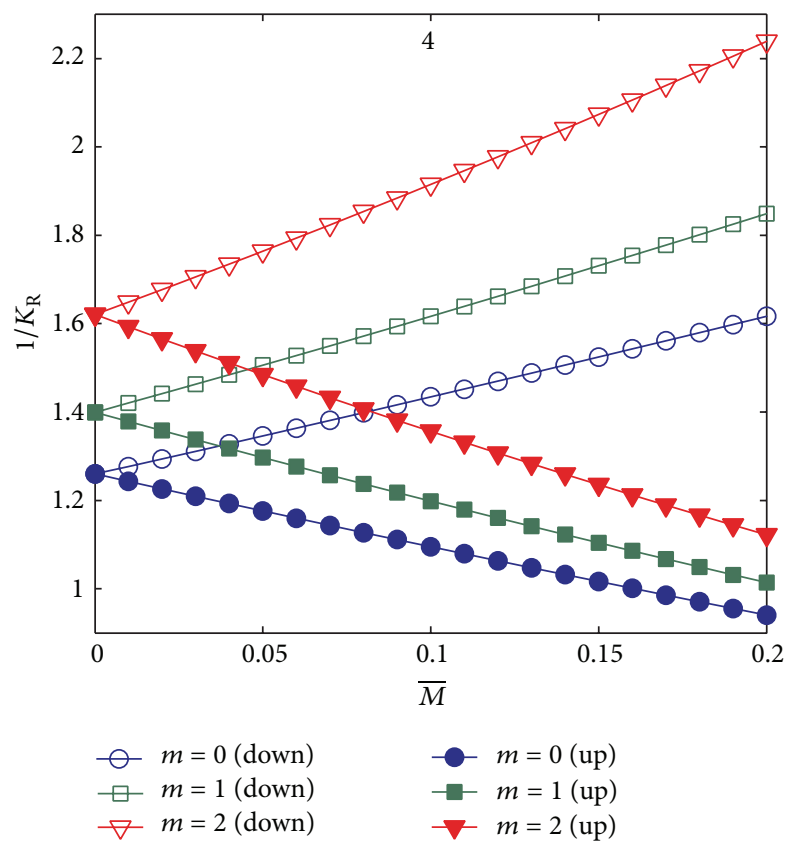

(d)

Figure 5: (Color online) Comparison of the relative phase velocity of the first four axial acoustic modes among the three circumferential modes in the turbulent flow confined by the lined-walled pipeline.

the three circumferential modes as functions of the average Mach number, it can be learned that the shear effect shows the most significant influence on the axisymmetric mode $(m=0)$. In the case of higher axial modes (Figures 4, 5(b), 5(c), and 5(d)), the influence of shear flow behaves similarly as shown in Figure 2. Comparison among the three higher axial modes reveals that the influence of wall impedance tends to channel the wave mode propagate near the wall. Such tendency is more obvious for a higher axial mode wave as shown in Figures $4(\mathrm{~d})$ and $5(d)$.

\subsection{Acoustic Attenuation}

4.2.1. Comparison between Laminar and Turbulent Flow. In this subsection, the attenuation coefficient due to the acoustic impedance at the wall is investigated. Specifically, Figure 6 compares the attenuation coefficient of the first four axial modes between the laminar ("lam") and turbulent ("tur") flow profiles in both the downstream (Figure 6(a)) and upstream (Figure 6(b)) propagations. The circumferential wave mode is assumed to be $m=0$; then the feature of the axisymmetric acoustic wave is addressed. 


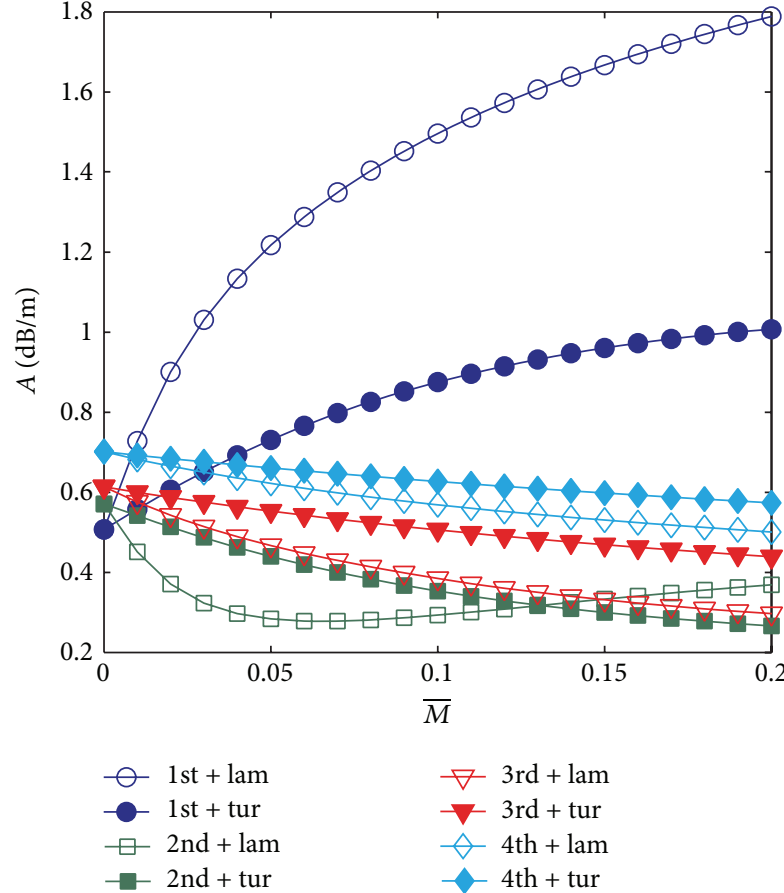

(a)

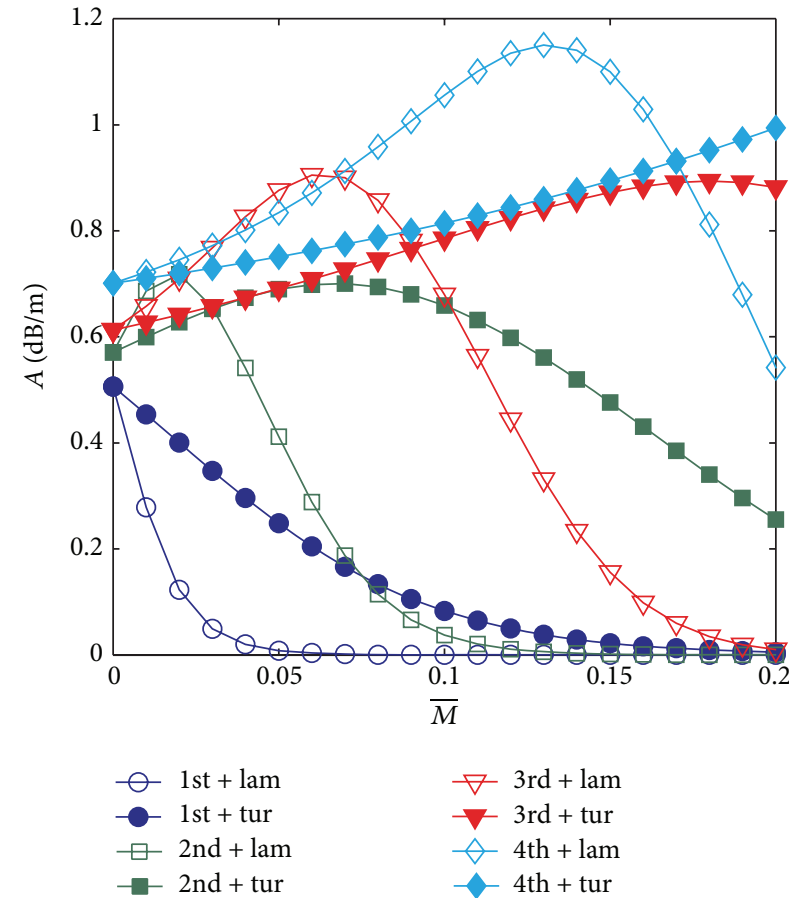

(b)

Figure 6: (Color online) Comparison of the attenuation coefficient of the first four axisymmetric modes ("1st," "2nd," "3rd," and "4th") propagating in the downstream (a) and upstream (b) directions in the laminar ("lam") and turbulent ("tur") velocity profiles.

In the absence of a moving fluid $(\bar{M}=0$ in Figures 6 (a) and 6(b)), a higher axial mode leads to a larger attenuation coefficient. As shown in Section 4.1.1, the relative phase velocity of a higher axial mode is larger which corresponds to shorter wavelength, steeper waveform, and larger attenuation. In the presence of a flow, the behavior of attenuation coefficient shows significant differences between the first axial mode and the other three axial modes.

For the first axial mode propagating in the downstream direction (Figure 6(a)), the attenuation coefficient becomes large in both the laminar and turbulent flows with the ascent of the average Mach number. Meanwhile, the ascending rate of attenuation coefficient in the laminar flow is quicker than that in the turbulent flow. As described in Figure 3, the shear effect regulates the first axial mode to a narrow layer near the wall in the downstream propagation. Then, the wave damping due to the acoustic impedance becomes larger in the presence of a higher Mach number. As the shear effect in the laminar flow is more noticeable, the wave attenuation in the laminar flow behaves severer. In the upstream propagation (Figure 6(b)), the attenuation coefficient slows down against the average Mach number in the laminar and turbulent flow. The attenuation descent is quicker in the laminar flow than that in turbulent flow. As the shear effect channels the acoustic wave in the upstream propagation into a narrow layer next to the pipeline center, the wave damping at the wall becomes slight. As the shear effect in the laminar flow is more significant than that in the turbulent flow, the decrease ratio of wave attenuation becomes quicker in the laminar flow.
Comparison of attenuation coefficients among the three high modes propagating in the downstream direction (Figure 6(a)) shows that the flow convection eases the wave attenuation, but the shear flow complicates the tendency of attenuation coefficients. In the upstream propagation, the flow convection slows down the propagation speed; then the occurrence probability of the wave damping at the wall increases, leading to an ascending of the attenuation coefficient. On the other hand, the shear effect regulates the high acoustic wave in the upstream propagation to a narrow layer near the pipeline center, leading to a decreasing of the wave damping at the wall. As the shear effect in the laminar flow is stronger than that in the turbulent flow, the average Mach number corresponding to the decrease of wave attenuation is smaller in the laminar flow. It should be noticed that the shear influence on a higher acoustic mode is less significant as the higher acoustic mode leads to a more complex propagation path.

\subsubsection{Comparison among Different Circumferential Modes.} Figure 7 compares the attenuation coefficients of the first four axial wave modes among the first three circumferential modes ( $m=0, m=1$, and $m=2$ ) in the laminar flow while Figure 8 displays the corresponding comparison in the turbulent flow.

In the absence of a shear flow, the attenuation coefficient of a higher circumferential mode is larger for each axial mode. The difference of the attenuation coefficients among the three 


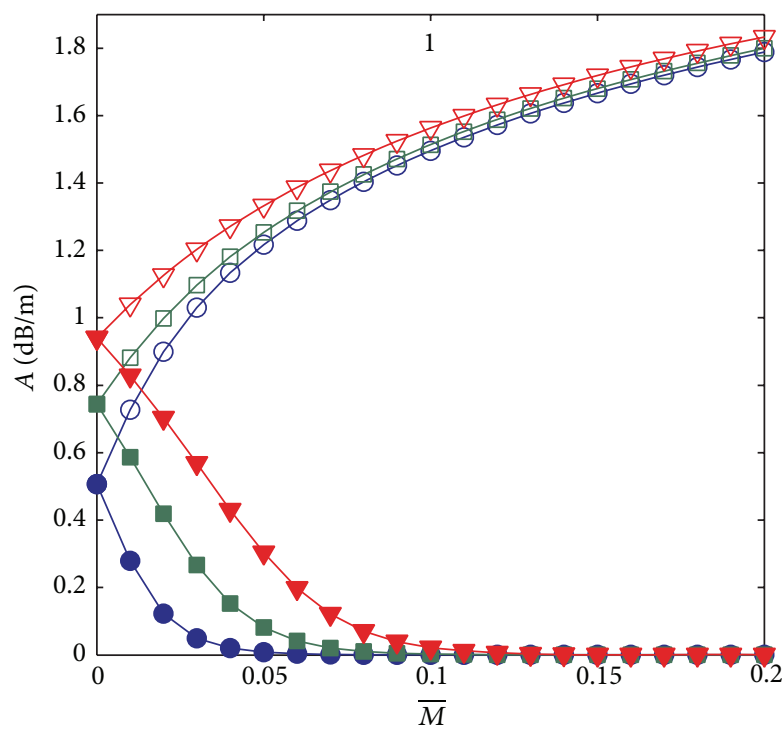

(a)

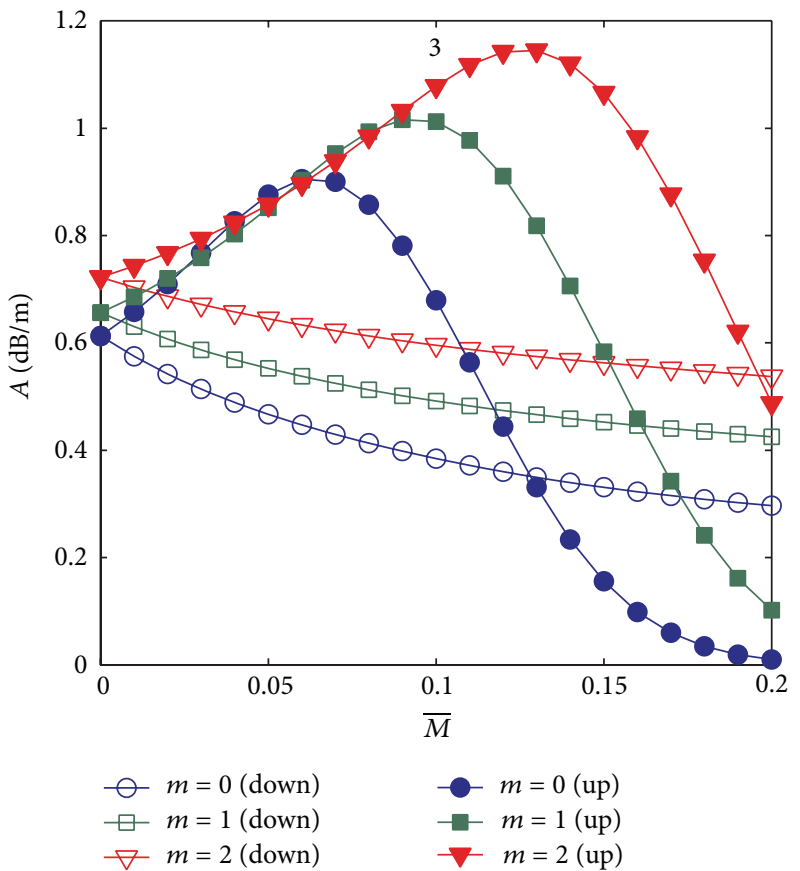

(c)

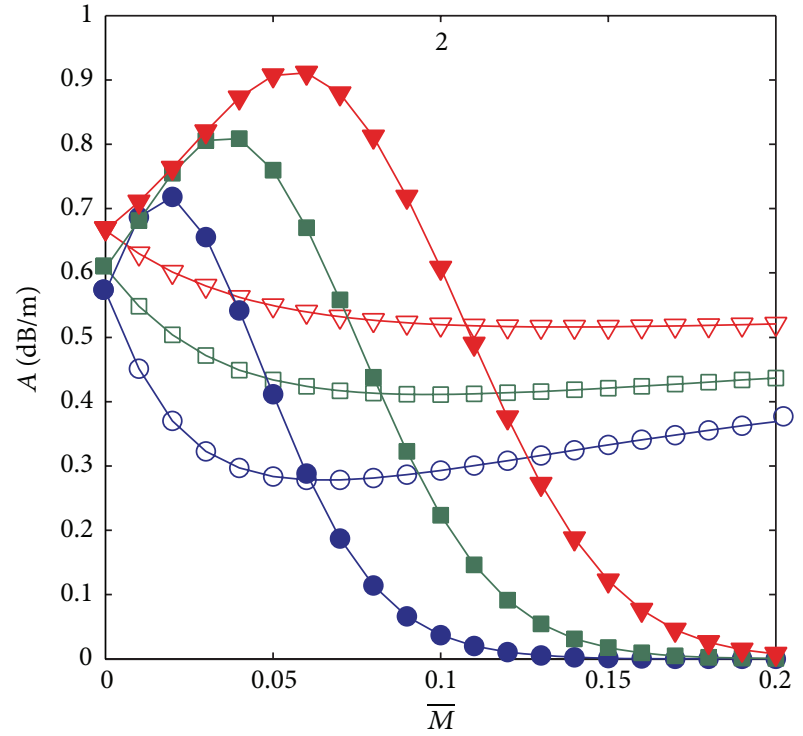

(b)

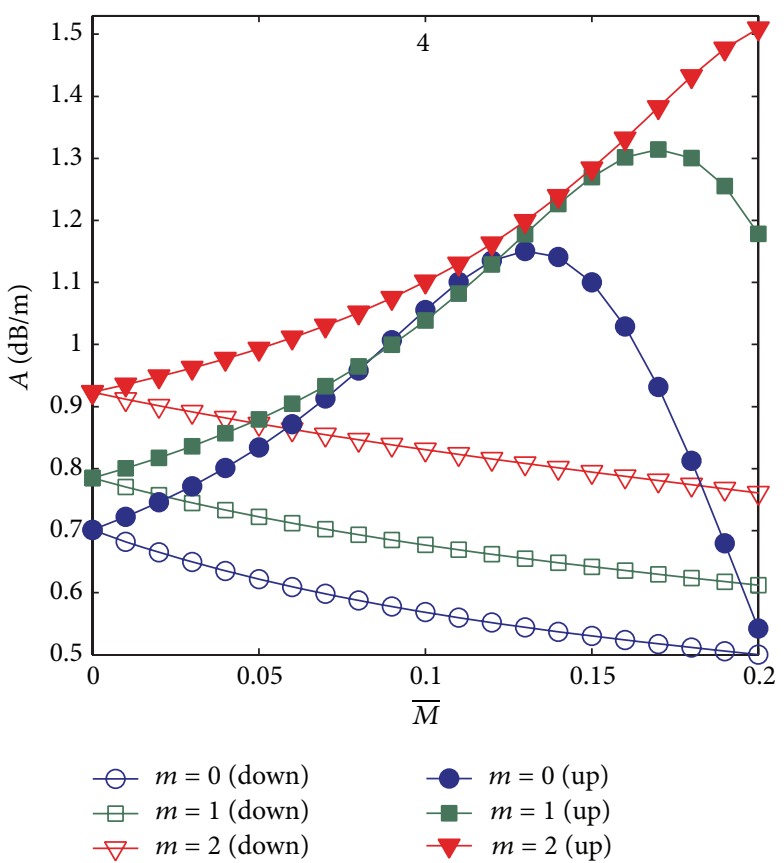

(d)

FIgure 7: (Color online) Comparison of the attenuation coefficients of the first four axial pseudo-modes among the three circumferential modes in the laminar flow confined by a lined-walled pipeline.

circumferential modes becomes more visible in the case of a higher axial mode. However, such tendency is not suitable for the first axial mode.

In the presence of a shear flow, the attenuation coefficient changes due to the existence of the shear and convective effects. Specifically, the influences of shear and convective actions on the other two circumferential modes $(m=1$ and $m=2$ ) are similar to the case of the axisymmetric mode $(m=0)$. It should be noticed that the shear effect on the attenuation coefficient of the axisymmetric acoustic mode shows more sensitivity compared with the case of the other two circumferential modes.

\section{Performance of Ultrasonic Flow Measurement}

In this section, the measurement performance of an ultrasonic flow meter based on the wave propagation is analyzed theoretically. To describe the measurement performance 


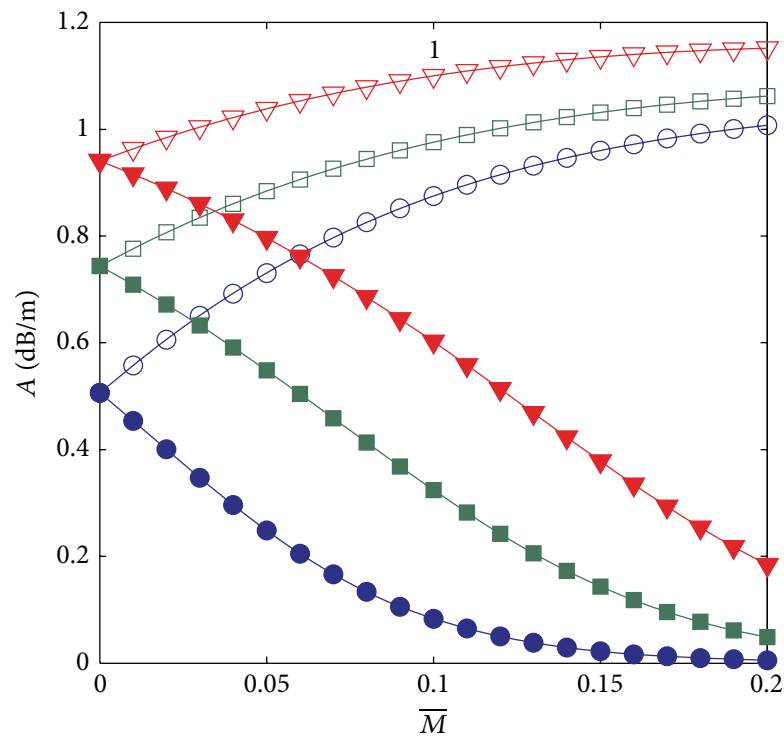

(a)

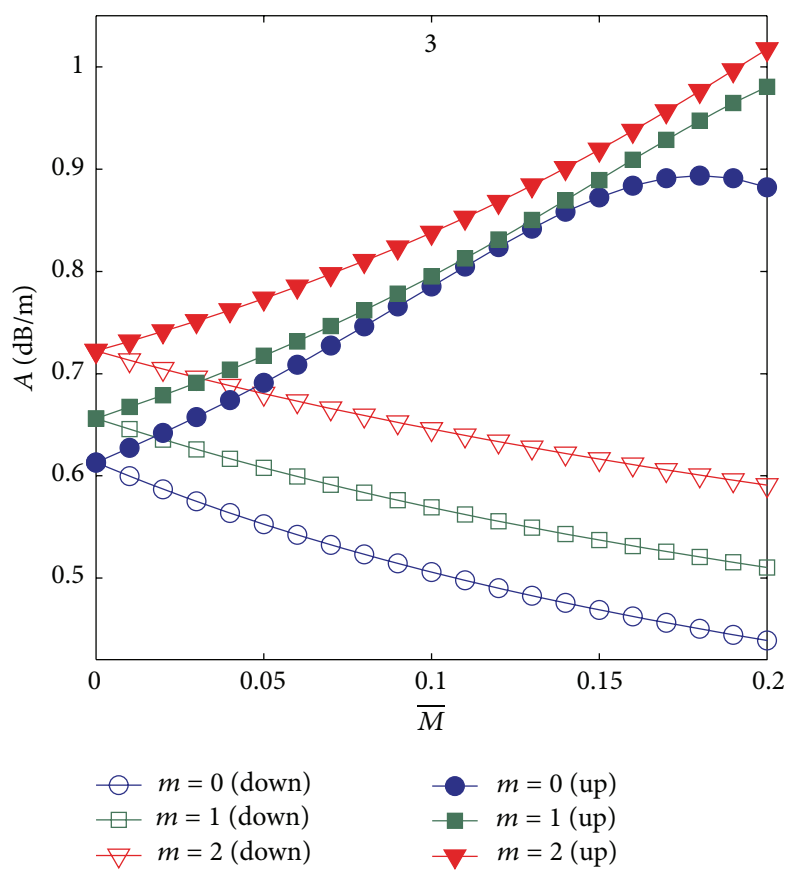

(c)

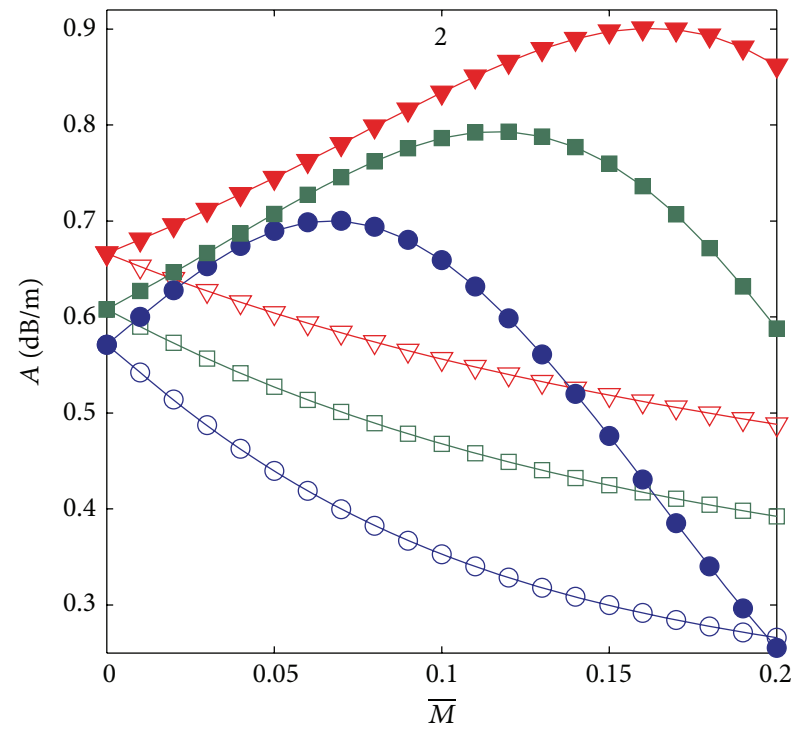

(b)

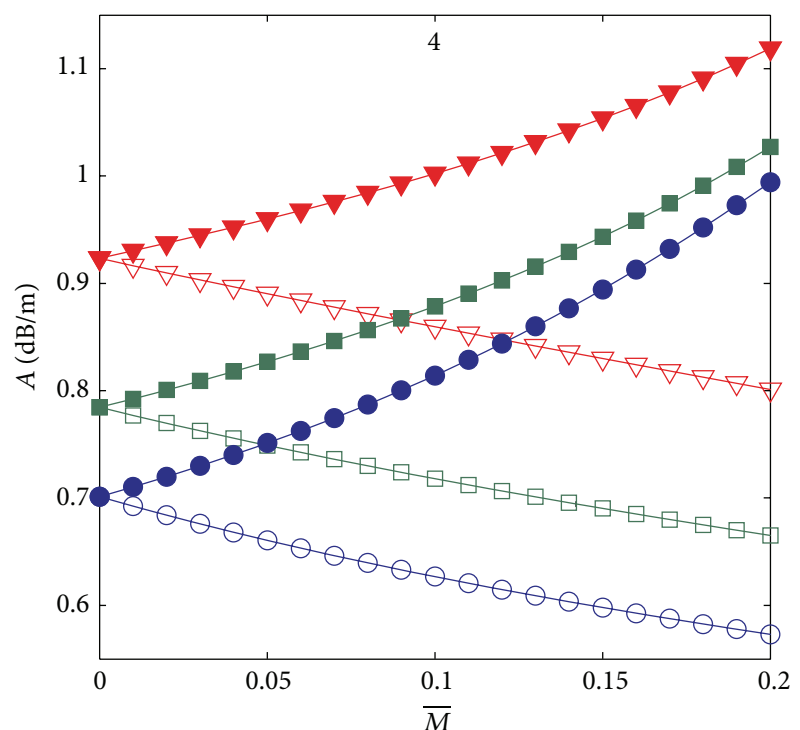

$$
\begin{array}{ll}
\square m=0 \text { (down) } & -m=0 \text { (up) } \\
\square m=1 \text { (down) } & -m=1 \text { (up) } \\
\nabla m=2 \text { (down) } & \square m=2 \text { (up) }
\end{array}
$$

(d)

FIgURE 8: (Color online) Comparison of the attenuation coefficients of the first four axial pseudo-modes among the three circumferential modes in the turbulent flow confined by a lined-walled pipeline.

explicitly, the measurement error is firstly defined $[5,19,22]$. Specifically, when a plane wave propagates in an inviscid fluid with a uniform flow $\left(\bar{c}_{0} \bar{M}\right)$, travelling a distance $L$ in the downstream direction requires

$$
t_{\text {down }}=\frac{L}{\bar{c}_{0}(1+\bar{M})},
$$

while the consumed time in the upstream direction is

$$
t_{\text {up }}=\frac{L}{\bar{c}_{0}(1-\bar{M})} \text {. }
$$

Subtracting (23) from (24) gives

$$
t_{\text {up }}-t_{\text {down }}=\frac{L}{\bar{c}_{0}(1-\bar{M})}-\frac{L}{\bar{c}_{0}(1+\bar{M})}=\frac{2 \bar{M} L}{\bar{c}_{0}\left(1-\bar{M}^{2}\right)} .
$$




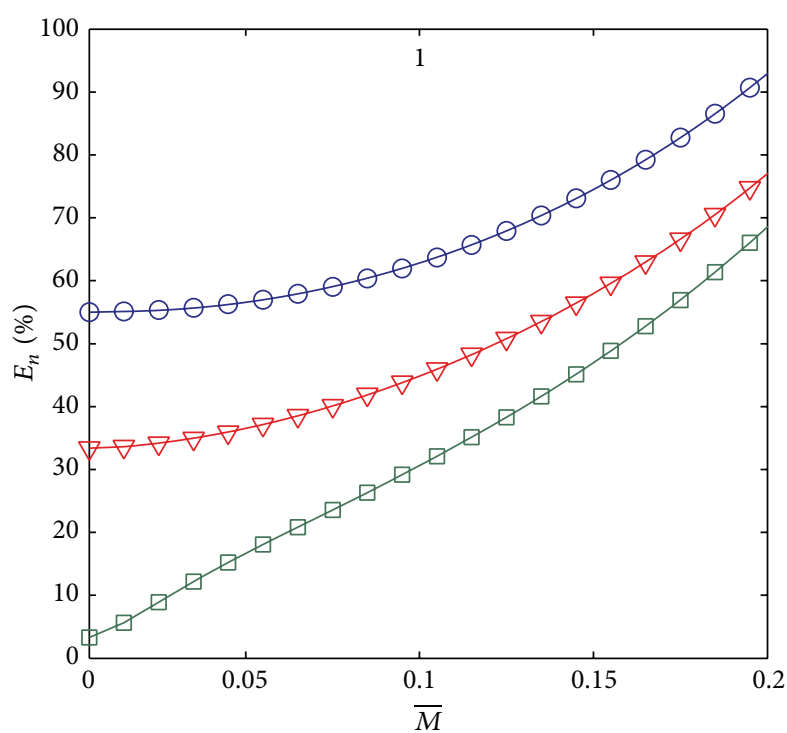

(a)

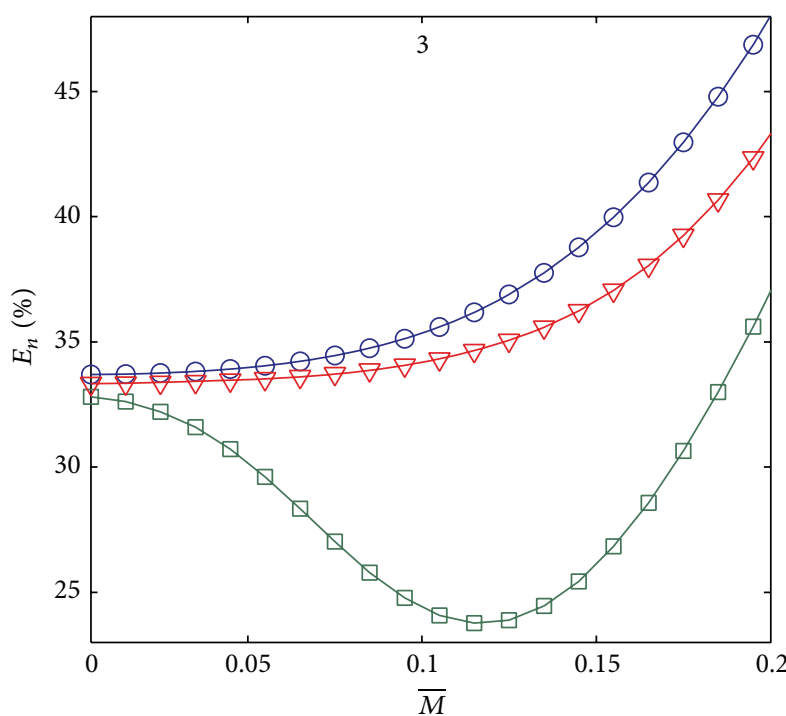

$\ominus$ Free
$\square$ Gen
$\nabla$ Rigid

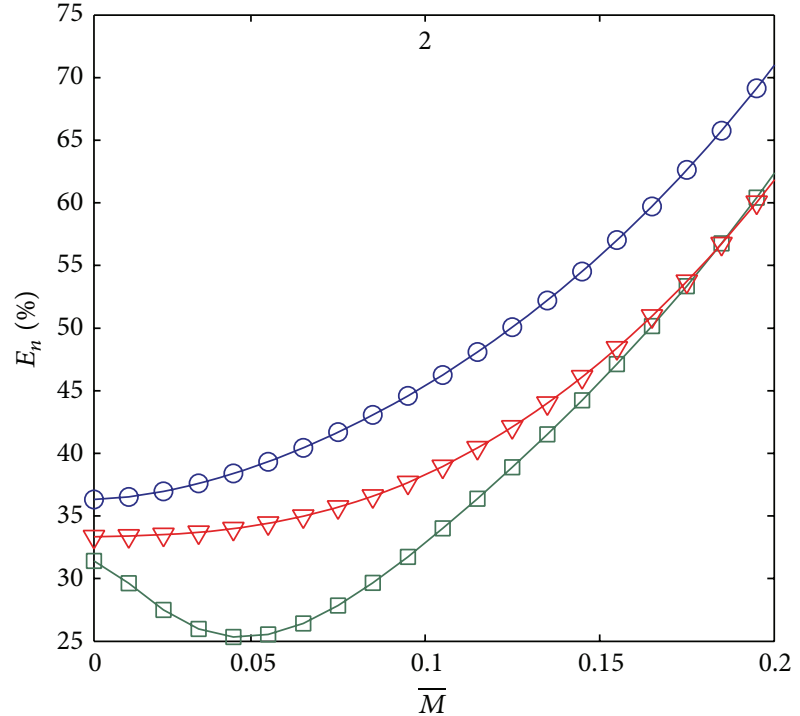

(b)

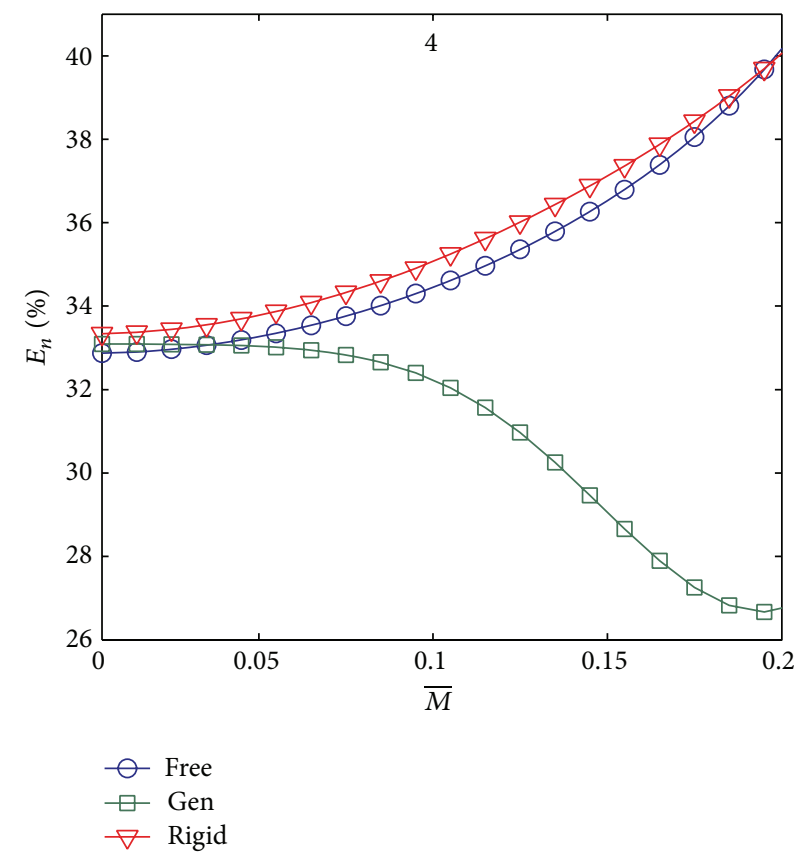

$$
\begin{aligned}
& \ominus \text { Free } \\
& \square \text { Gen } \\
& \nabla \text { Rigid }
\end{aligned}
$$

(d)

Figure 9: (Color online) Comparison of the measurement errors of the first four axial modes among the three different boundary configurations in the laminar flow. See Figure 2 for legend interpretation.

Under the assumption of $\bar{M}^{2} \ll 1$, one obtains

$$
t_{\text {up }}-t_{\text {down }}=\frac{2 L}{\bar{c}_{0}} \bar{M}
$$

and the volume flow $\left(Q_{V}\right)$ then can be obtained by

$$
Q_{V}=A_{P} \bar{M}=\frac{A_{P} \bar{c}_{0}}{2 L}\left(t_{\text {up }}-t_{\text {down }}\right)
$$

For a specific wave mode $(n)$, from (4), traveling a distance $L$ requires

$$
t_{\text {down }}^{n}=\frac{k_{0}\left(K_{\mathrm{R}}^{\text {down }}\right)^{n} L}{\omega}, \quad t_{\text {up }}^{n}=\frac{k_{0}\left(K_{\mathrm{R}}^{\mathrm{up}}\right)^{n} L}{\omega} .
$$

Then we have

$$
\begin{aligned}
t_{\text {up }}^{n}-t_{\text {down }}^{n} & =\frac{k_{0} L}{\omega}\left(\left(K_{\mathrm{R}}^{\mathrm{up}}\right)^{n}-\left(K_{\mathrm{R}}^{\text {down }}\right)^{n}\right) \\
& =\frac{L}{\bar{c}_{0}}\left(\left(K_{\mathrm{R}}^{\mathrm{up}}\right)^{n}-\left(K_{\mathrm{R}}^{\text {down }}\right)^{n}\right),
\end{aligned}
$$




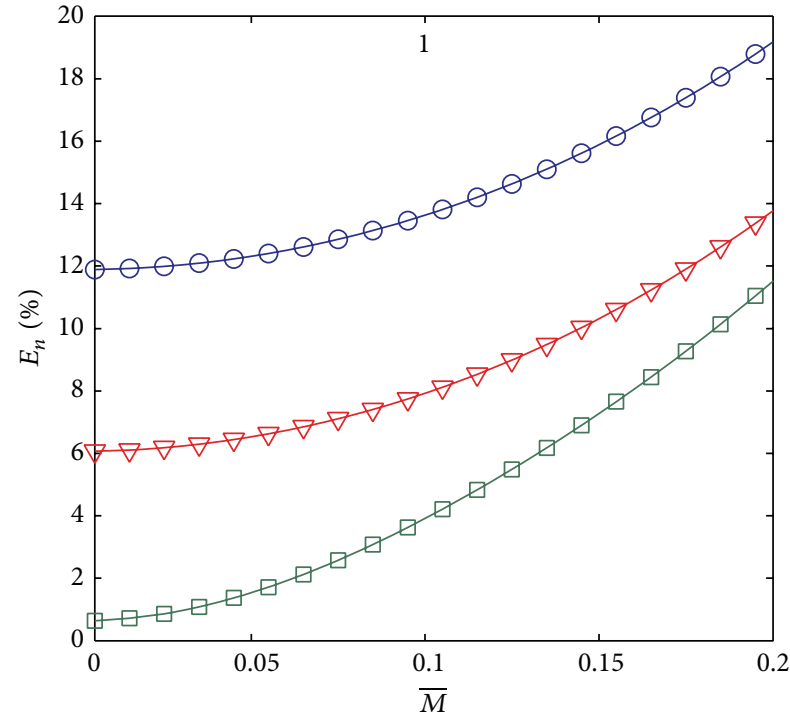

(a)

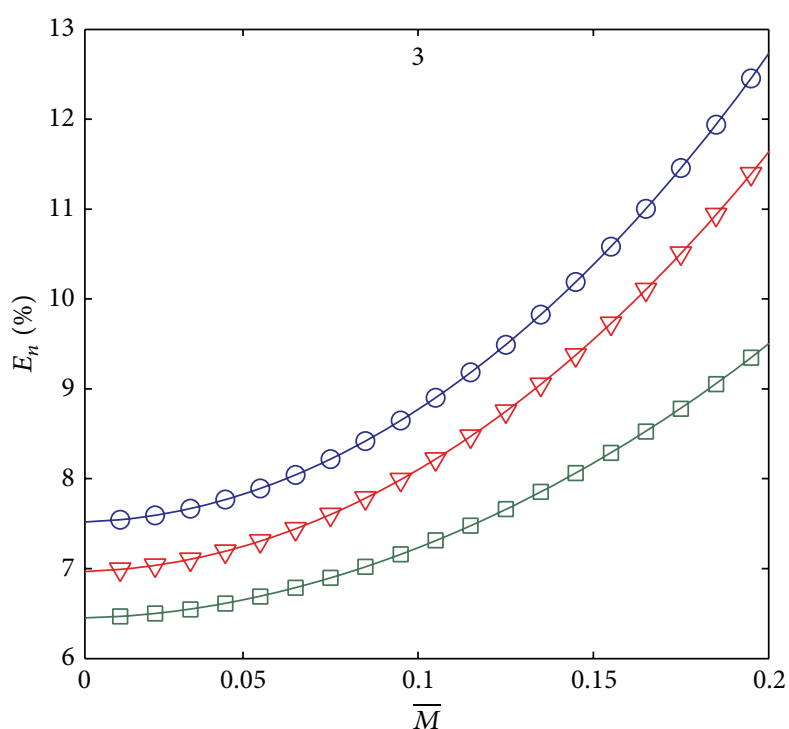

$\bigcirc$ Free

$\square$ Gen

$\nabla$ Rigid

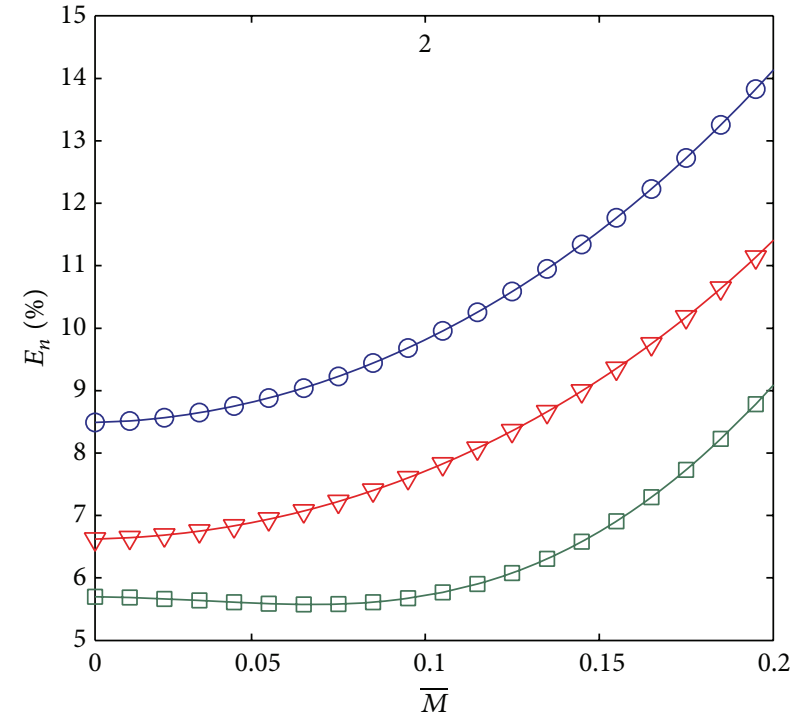

(b)

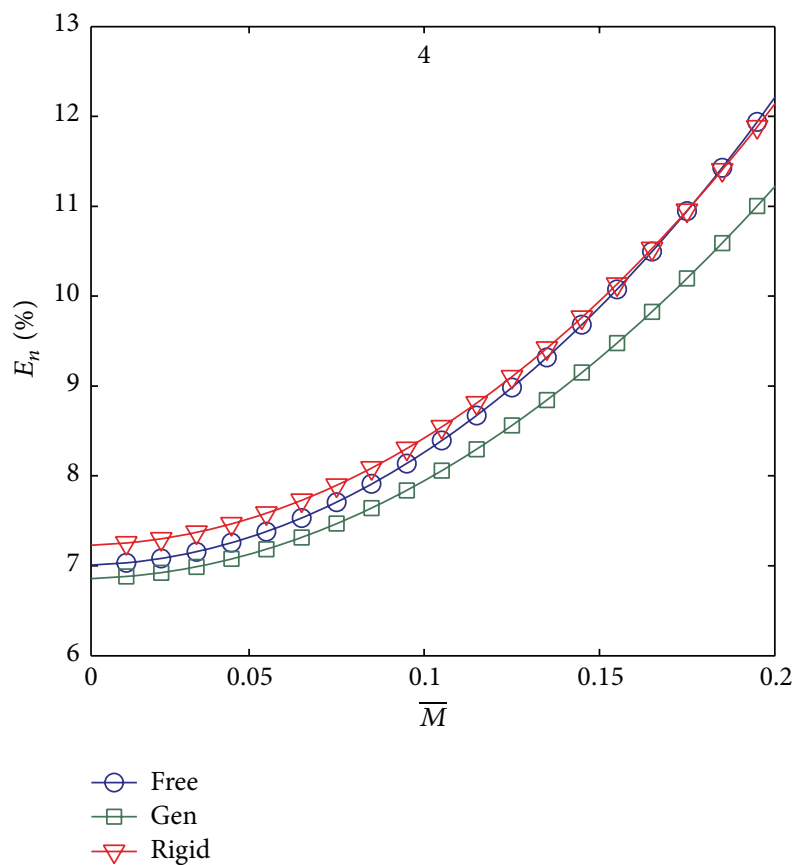

(d)

FIGURE 10: (Color online) Comparison of the measurement errors of the first four axial modes among the three different boundary configurations in the turbulent flow. See Figure 2 for legend interpretation.

and the corresponding measured volume flow is

$$
\begin{aligned}
Q_{V}^{n} & =\frac{A_{P} \bar{c}_{0}}{2 L}\left(t_{\text {up }}^{n}-t_{\text {down }}^{n}\right) \\
& =\frac{A_{P}}{2}\left(\left(K_{\mathrm{R}}^{\text {up }}\right)^{n}-\left(K_{\mathrm{R}}^{\text {down }}\right)^{n}\right) .
\end{aligned}
$$

Thus, the measurement error of a specific wave mode can be expressed by

$$
E_{n}=\frac{Q_{V}^{n}-Q_{V}}{Q_{V}}=\frac{\left(K_{\mathrm{R}}^{\mathrm{up}}\right)^{n}-\left(K_{\mathrm{R}}^{\text {down }}\right)^{n}}{2 \bar{M}}-1
$$

It can be inferred that the measurement error represents the symmetric characteristics of the real component of the axial wavenumber in the downstream and upstream propagations with respect to the case of the static fluid.

5.1. Comparison among Different Kinds of Boundary Condition. Figure 9 presents the difference of the measurement errors of the first four axial pseudo-modes among the three different boundary configurations (freeboundary/lined/rigid wall) in the laminar flow, while Figure 10 details the corresponding comparison in the 


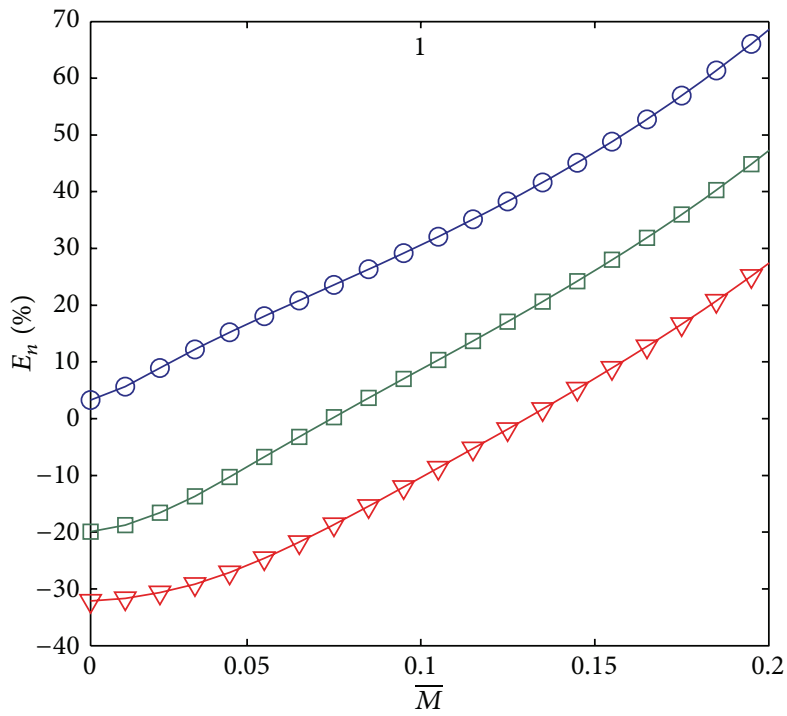

(a)

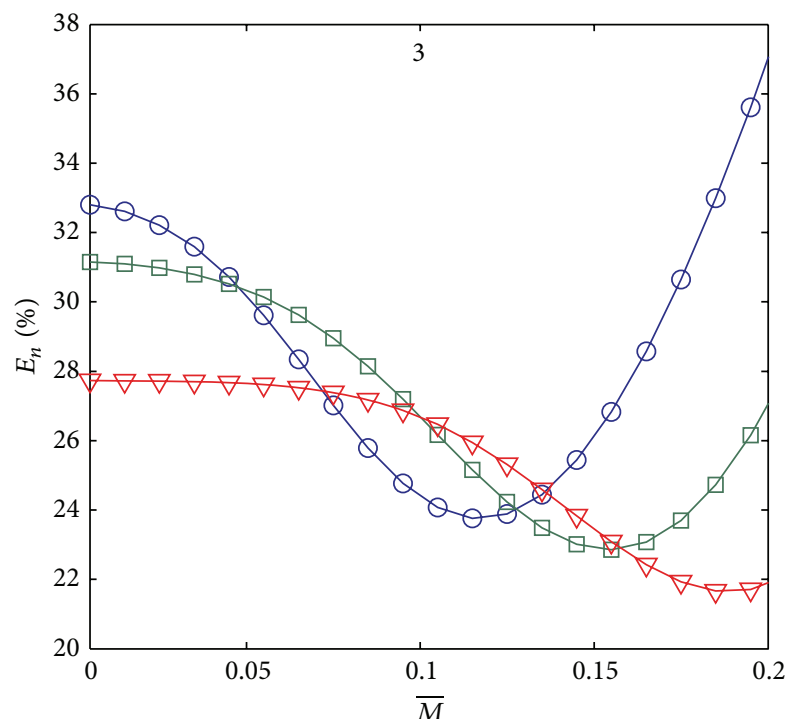

$\bigcirc m=0$
$\square m=1$
$\nabla m=2$

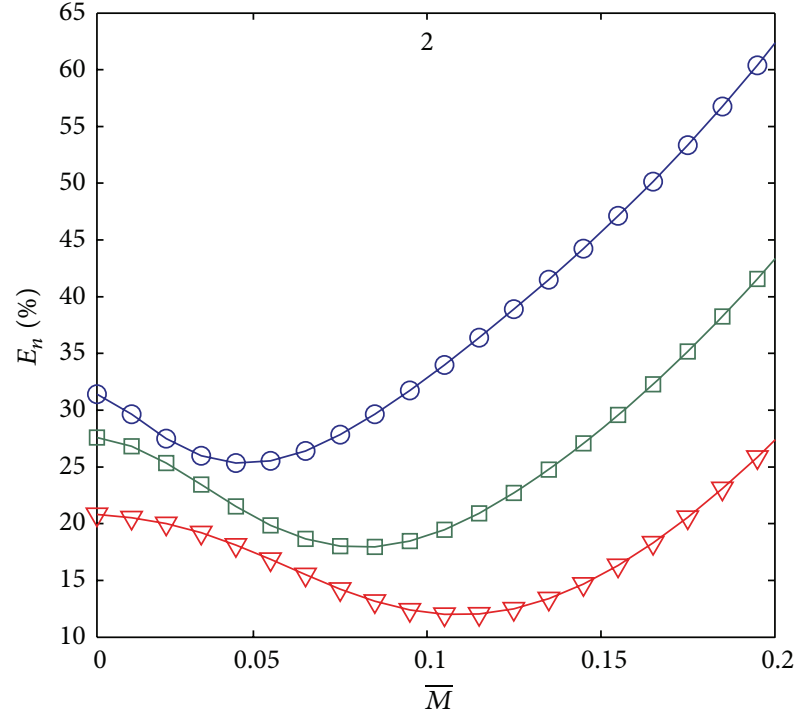

(b)

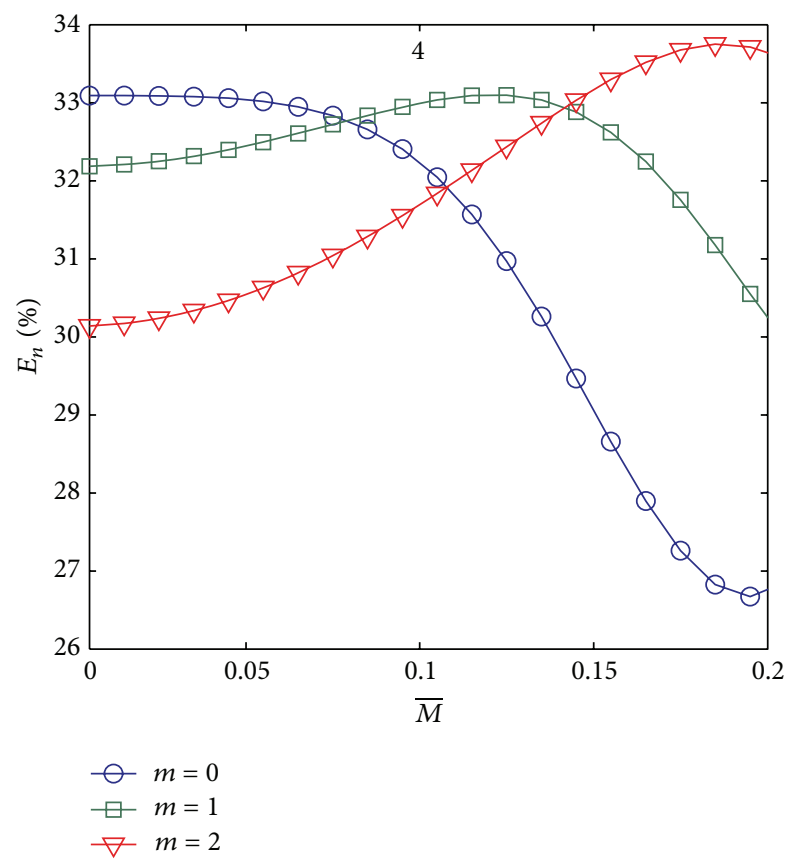

(d)

FIGURE 11: (Color online) Comparison of the measurement errors of the first four axial modes among the three circumferential modes $(m=0$, $m=1$, and $m=2$ ) in the laminar flow confined by the lined-walled pipeline.

turbulent flow. The circumferential mode is assumed to be $m=0$; thus the feature of the axisymmetric acoustic wave is analyzed. Obviously, the measurement error in the lined-walled pipeline is the smallest in the laminar and turbulent flow. Furthermore, the measurement error of each acoustic mode is higher in the laminar flow than that in the turbulent flow.

In the turbulent flow (Figure 10), the measurement error of each axial mode grows up semilinearly with the mean Mach number whatever the boundary condition is. As the influence of flow convection on the relative phase velocity in the downstream and upstream wave propagations is semisymmetric with respect to the case of the static fluid (Figure 3), the linearity of the measurement error in terms of the average Mach number is maintained. For a higher axial mode, the variation range of the measurement error with respect to the average Mach number becomes shorter. A possible interpretation may be that the complex reflection and refraction at the fluid-wall interface for the high acoustic mode result in a complicated propagation path in the pipeline. Then, the inconsistent effect of flow profile on the acoustic wave propagating in downstream and upstream 


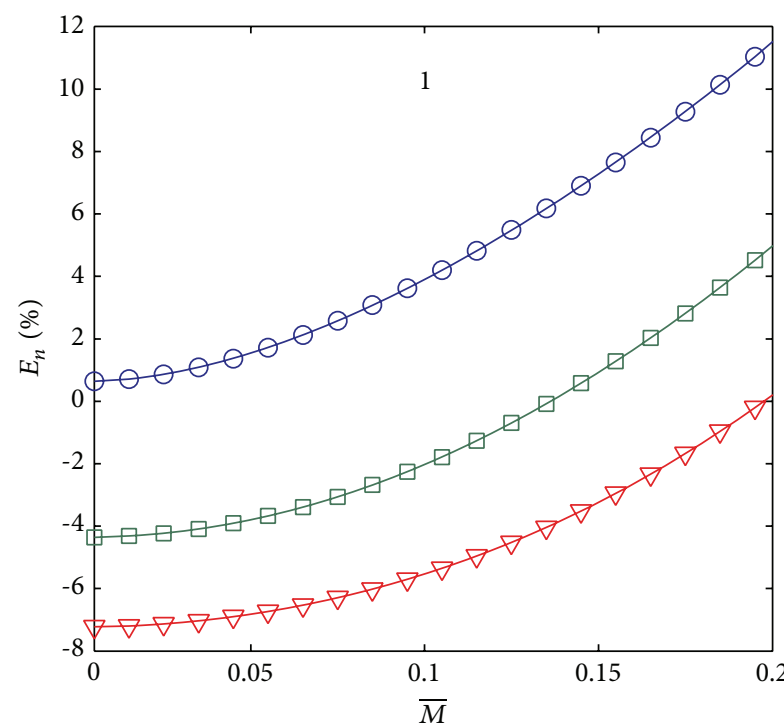

(a)

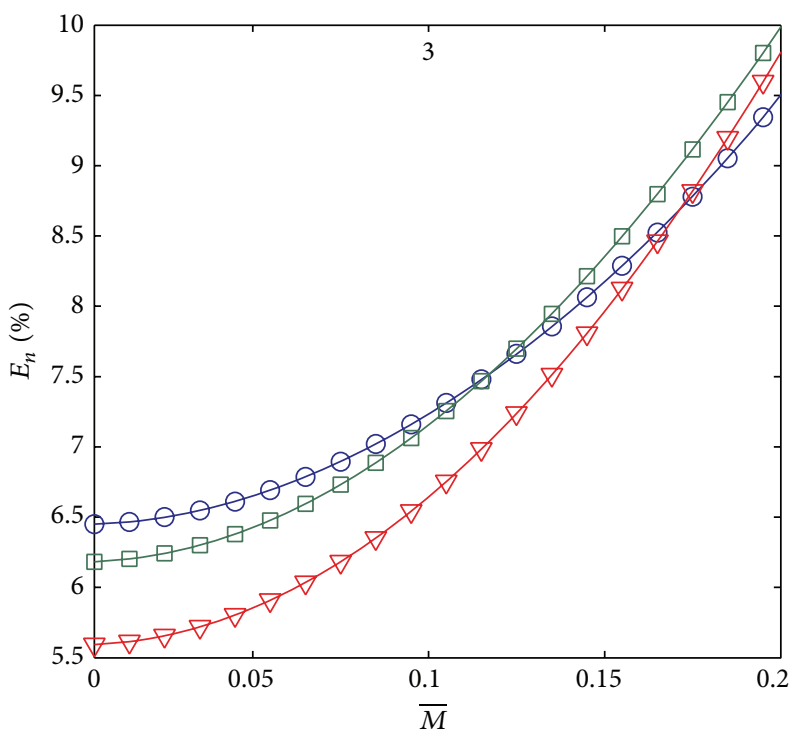

$\bigcirc-m=0$

$\square m=1$

$\nabla m=2$

(c)

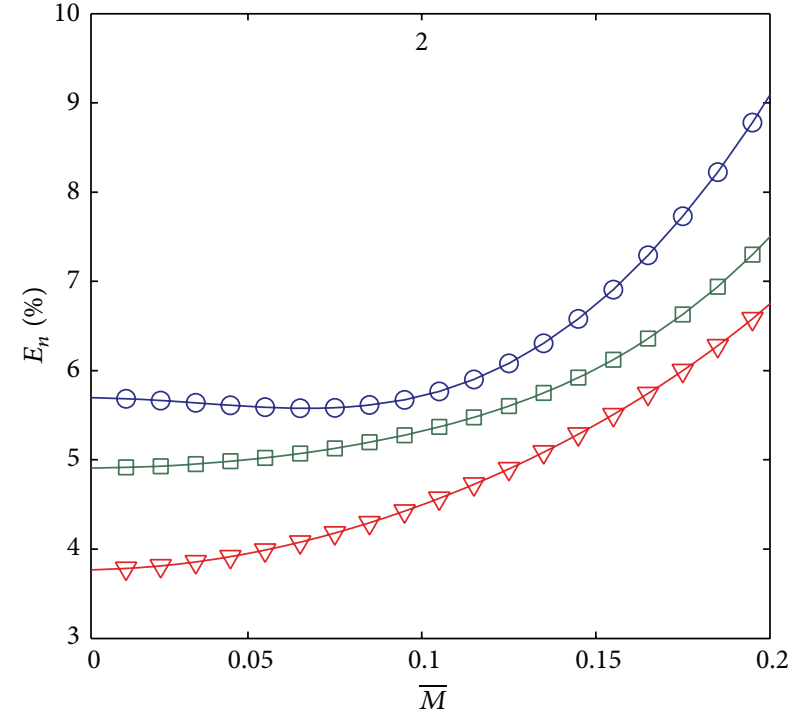

(b)

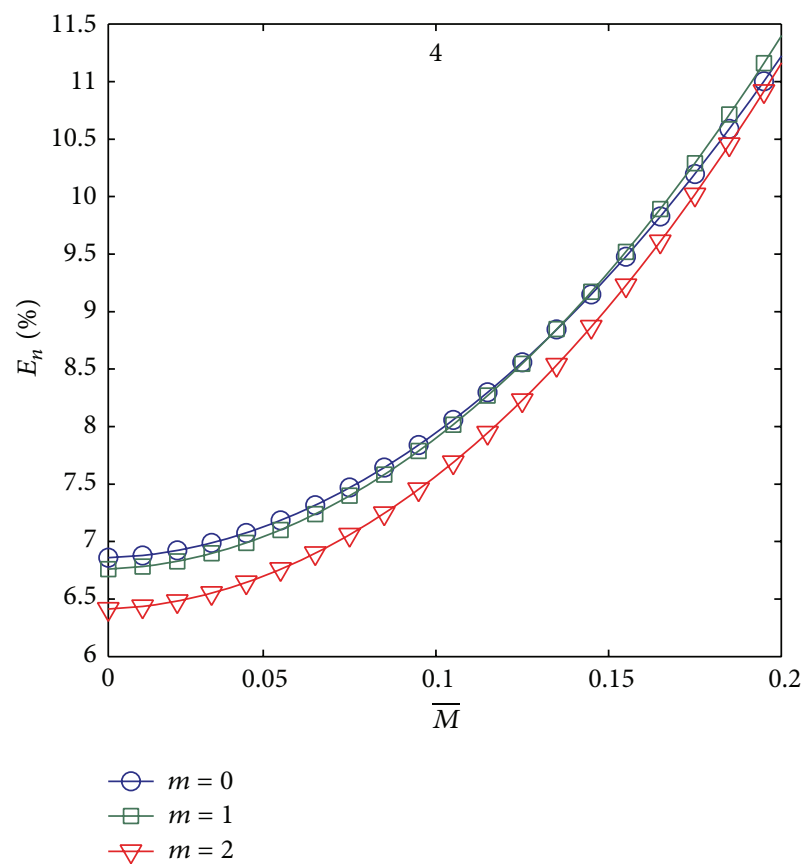

(d)

FIGURE 12: (Color online) Comparison of the measurement errors of the first four axial modes among the three circumferential modes in the turbulent flow confined by a lined-walled pipeline.

directions is compensated. Such tendency becomes more obvious in the laminar flow as shown in Figure 9.

Figure 9 reveals that a higher axial mode has a slower variation of the measurement error. Comparing with the measurement error in the turbulent flow shows that the shear effect sharply enlarges the measurement error. The complex propagation path of a higher axial mode due to the reflection and refraction compensates the shear effect on the measurement error. The mechanism of the acoustic impedance decreases the measurement error compared with the cases of the rigid and free-boundary configurations.
With the increase of the mean Mach number, the shear effect on the measurement error begins to play the dominant role which worsens the measurement performance. As a result, the measurement error in a lined-walled pipeline firstly decreases against the average Mach number but finally increases. The complex reflection and refraction ease the shear effect and then postpone the increase ratio of the measurement error. In the literature, Lechner [19] and Willatzen [20-24] did similar work in the case of very small Mach number. Their work reveals that the measurement error turns to be insensitive to the Mach number. From Figure 9, it can 
be learned that their contributions are consistent with the present study.

\subsection{Comparison among Different Circumferential Modes.} Figure 11 compares the measurement errors of the first four axial modes among the three circumferential modes in the laminar flow confined by the lined-walled pipeline and Figure 12 reveals the corresponding comparison in the turbulent flow.

Clearly, the shear and convective effects on the measurement performance of the two nonaxisymmetric modes $(m=$ 1 and $m=2$ ) are similar to the case of the axisymmetric mode $(m=0)$. Comparison among the three circumferential modes reveals that the measurement error of the axisymmetric mode is the most sensitive. For each axial mode, the measurement error of a lower circumferential mode is larger. However, such tendency is not suitable when the Mach number is large.

In the end, it can be learned that the effect of flow convection on the phase velocity in the downstream and upstream propagations keeps a symmetric way which can be inferred from Figures 3 and 5. In the presence of a shear flow, the inconsistency of phase velocity between the downstream and upstream propagations is obvious as shown by Figures 2 and 4 . The shear effect severely alters the influence of flow convection. It should be pointed out that in many industrial applications, the actual flow Mach number may be small. Then the measurement error of each mode in the laminar and turbulent flow changes semilinearly with respect to the average Mach number. Furthermore, with the increase of the flow Mach number, the turbulent flow profile seems to be a better candidate to describe the actual condition than the laminar flow.

\section{Conclusion}

In this paper, the nonaxisymmetric wave propagation in the inviscid fluid with a shear mean flow confined by a circular pipeline is studied while the acoustic wave is assumed to be isentropic and linear. The mathematical formulation is deduced into the form of a second-order differential equation with respect to the acoustic pressure. A general boundary condition is presented to handle three different configurations with free-boundary, rigid, and lined walls respectively. Using the Fourier-Bessel theory, a method is provided to numerically calculate the axial wavenumber, which describes the phase velocity and wave attenuation. Numerical analysis reveals the following.

(1) The phase velocity of wave propagation in the rigidwalled pipeline turns out to be the largest among the three types of boundary condition while the phase velocity in the lined-walled pipeline is the smallest.

(2) Wave propagation with a higher circumferential mode has a larger phase velocity. In the case of a higher axial mode, the distinction of the phase velocity among the circumferential modes is more explicit.
(3) The effect of flow convection accelerates the wave propagation in the downstream direction and decelerates the wave propagation in the upstream direction. Meanwhile, the corresponding attenuation coefficient increases in the upstream propagation and decreases in the downstream propagation. However, the shear effect greatly alters the influence of flow convection on both phase velocity and attenuation coefficient.

(4) Acoustic wave with a higher circumferential mode results in severer wave attenuation. Furthermore, a high circumferential mode postpones the shear effect on the wave attenuation.

In the end, the measurement performance of an ultrasonic flow meter is numerically investigated. The effects of circumferential mode, boundary condition, and shear profile are parametrically demonstrated.

\section{Conflict of Interests}

The authors declare that there is no conflict of interests regarding the publication of this paper.

\section{Acknowledgments}

The work described in this paper is funded by the National Natural Science Foundation of China (nos. 91216201 and 51205403). The authors gratefully acknowledge the funding.

\section{References}

[1] L. M. B. C. Campos and J. M. G. S. Oliveira, "On the acoustic modes in a duct containing a parabolic shear flow," Journal of Sound and Vibration, vol. 330, no. 6, pp. 1166-1195, 2011.

[2] R. Boucheron, H. Bailliet, and J.-C. Valiere, "Analytical solution of multimodal acoustic propagation in circular ducts with laminar mean flow profile," Journal of Sound and Vibration, vol. 292, no. 3-5, pp. 504-518, 2006.

[3] S. W. Rienstra and M. Darau, "Boundary-layer thickness effects of the hydrodynamic instability along an impedance wall," Journal of Fluid Mechanics, vol. 671, pp. 559-573, 2011.

[4] G. G. Vilenski and S. W. Rienstra, "On hydrodynamic and acoustic modes in a ducted shear flow with wall lining," Journal of Fluid Mechanics, vol. 583, pp. 45-70, 2007.

[5] Y. Chen, Y. Huang, and X. Chen, "Acoustic propagation in viscous fluid with uniform flow and a novel design methodology for ultrasonic flow meter," Ultrasonics, vol. 53, no. 2, pp. 595606, 2013.

[6] Y. Chen, Y. Huang, and X. Chen, “Thermal-mechanical wave propagation in inviscid non-uniform flow confined by heating pipeline and implications for transit-time flow meter," Acta Acustica United with Acustica, vol. 99, no. 4, pp. 503-513, 2013.

[7] D. C. Pridmore-Brown, "Sound propagation in a fluid flowing through an attenuating duct," Journal of Fluid Mechanics, vol. 4, no. 4, pp. 393-406, 1958.

[8] P. Mungur and H. E. Plumblee, "Propagation and attenuation of sound in a soft walled annular duct containing a sheared flow," NASA Report SP-207, 1969. 
[9] G. R. Gogate and M. L. Munjal, "Sound propagation in ducts with bulk reacting lining in the presence of laminar mean flow," The Journal of the Acoustical Society of America, vol. 99, no. 3, pp. 1779-1782, 1996.

[10] S.-H. Ko, "New approach to the solution of eigenvalue problems in circular flow ducts (a Taylor series method)," The Journal of the Acoustical Society of America, vol. 70, no. 1, pp. 205-212, 1981.

[11] W. Eversman, "Initial values for the integration scheme to compute the eigenvalues for propagation in ducts," Journal of Sound and Vibration, vol. 50, no. 1, pp. 159-162, 1977.

[12] N. K. Agarwal and M. K. Bull, "Acoustic wave propagation in a pipe with fully developed turbulent flow," Journal of Sound and Vibration, vol. 132, no. 2, pp. 275-298, 1989.

[13] V. Pagneux and B. Froelich, "Influence of low mach number shear flow on acoustic propagation in ducts," Journal of Sound and Vibration, vol. 246, no. 1, pp. 137-155, 2001.

[14] S. W. Rienstra, "A classification of duct modes based on surface waves," Wave Motion, vol. 37, no. 2, pp. 119-135, 2003.

[15] A. F. Smith, N. C. Ovenden, and R. I. Bowles, "Flow and geometry induced scattering of high frequency acoustic duct modes," Wave Motion, vol. 49, no. 1, pp. 109-124, 2012.

[16] E. J. Brambley, A. M. J. Davis, and N. Peake, "Eigenmodes of lined flow ducts with rigid splices," Journal of Fluid Mechanics, vol. 690, pp. 399-425, 2012.

[17] C. Buske, C. Richter, F. Thiele, C. Yu, and M. Zhuang, "Validation of a zonal approach computing the sound radiation from lined ducts," AIAA Journal, vol. 48, no. 12, pp. 2899-2908, 2010.

[18] D. Marx, Y. Aurégan, H. Bailliet, and J.-C. Valière, "PIV and LDV evidence of hydrodynamic instability over a liner in a duct with flow," Journal of Sound and Vibration, vol. 329, no. 18, pp. 3798-3812, 2010.

[19] H. Lechner, "Ultrasonic flow metering based on transit time differentials which are insensitive to flow profile," The Journal of the Acoustical Society of America, vol. 74, no. 3, pp. 955-959, 1983.

[20] M. Willatzen, "Comments on 'ultrasonic flow metering based on transit time differentials which are insensitive to flow profile,' The Journal of the Acoustical Society of America, vol. 107, no. 3, pp. 1762-1765, 2000.

[21] M. Willatzen, "Sound propagation in a moving fluid confined by cylindrical walls-a comparison between an exact analysis and the local-plane-wave approximation," Journal of Sound and Vibration, vol. 247, no. 4, pp. 719-729, 2001.

[22] M. Willatzen, "Flow acoustics modelling and implications for ultrasonic flow measurement based on the transit-time method," Ultrasonics, vol. 41, no. 10, pp. 805-810, 2004.

[23] M. Willatzen, "Ultrasonic flow measurement and wall acoustic impedance effects," Ultrasonics, vol. 41, no. 9, pp. 719-726, 2004.

[24] M. Willatzen, "Perturbation theory applied to sound propagation in flowing media confined by a cylindrical waveguide," The Journal of the Acoustical Society of America, vol. 109, no. 1, pp. 102-107, 2001.

[25] W. J. Jacobi, "Propagation of sound waves along liquid cylinders," The Journal of the Acoustical Society of America, vol. 21, pp. 120-127, 1949.

[26] T. C. Lin and G. W. Morgan, "Wave propagation through fluid contained in a cylindrical, elastic shell," The Journal of the Acoustical Society of America, vol. 28, pp. 1165-1176, 1956.

[27] G. N. Watson, A Treatise on the Theory of Bessel Functions, Cambridge University Press, London, UK, 2nd edition, 1966.
[28] B. G. Korenev, Bessel Functions and Their Applications, Taylor and Franics, London, UK, 2002.

[29] Y. Chen, Y. Huang, and X. Chen, "Fourier-bessel theory on flow acoustics in inviscid shear pipeline fluid flow," Communications in Nonlinear Science and Numerical Simulation, vol. 18, no. 11, pp. 3023-3035, 2013.

[30] Y. Chen, Y. Huang, and X. Chen, "Ultrasonic wave propagation in thermoviscous moving fluid confined by heating pipeline and flow measurement performance," The Journal of the Acoustical Society of America, vol. 134, no. 3, pp. 1863-1874, 2013.

[31] Y. Chen, Y. Huang, Y. Zhao, L. Cao, and X. Chen, "Bessel-fourier theory for acoustic propagation in inviscid fluid flow confined by rigid cylindrical waveguide," in Proceedings of the American Control Conference (ACC '13), pp. 2112-2117, Washington, DC, USA, June 2013. 

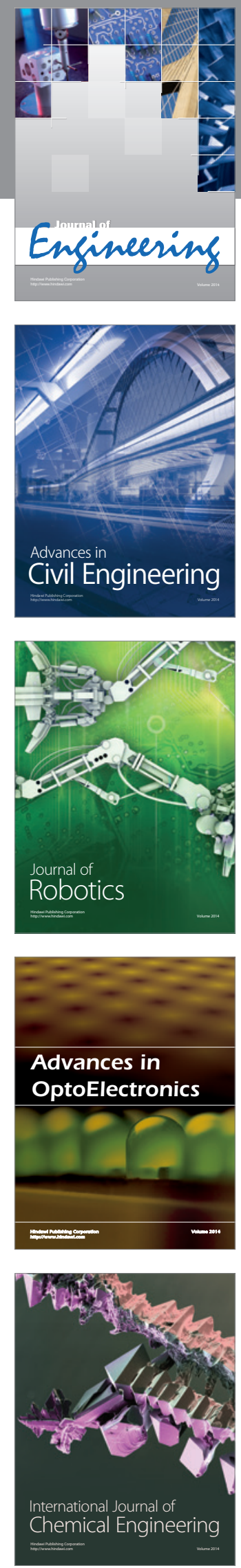

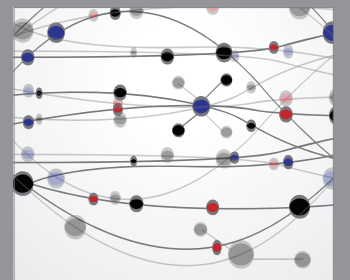

The Scientific World Journal
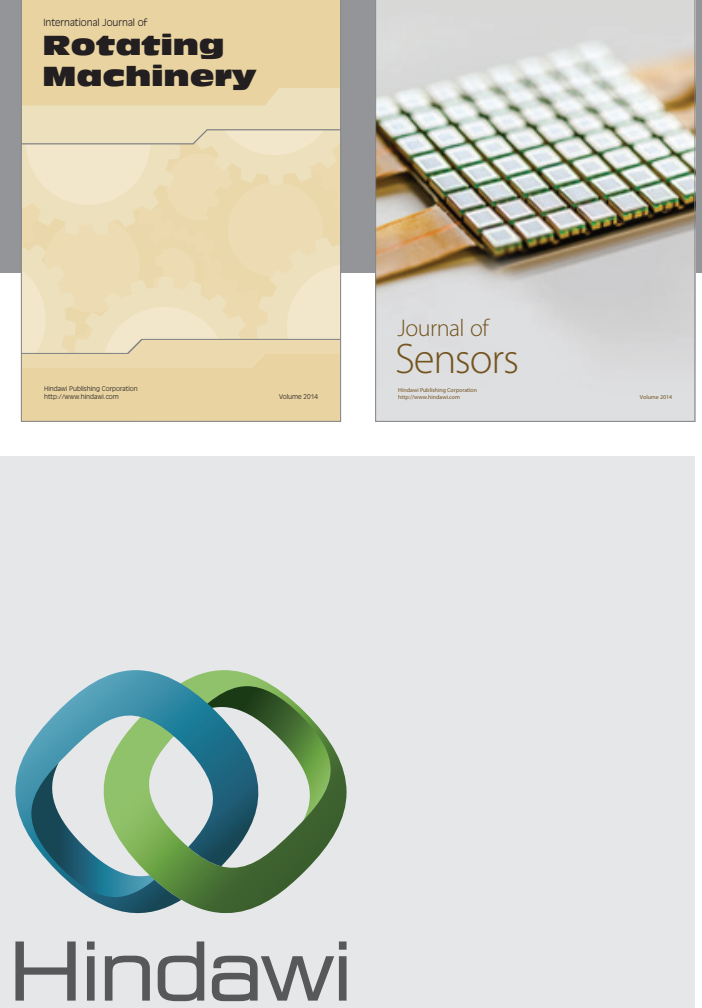

Submit your manuscripts at http://www.hindawi.com
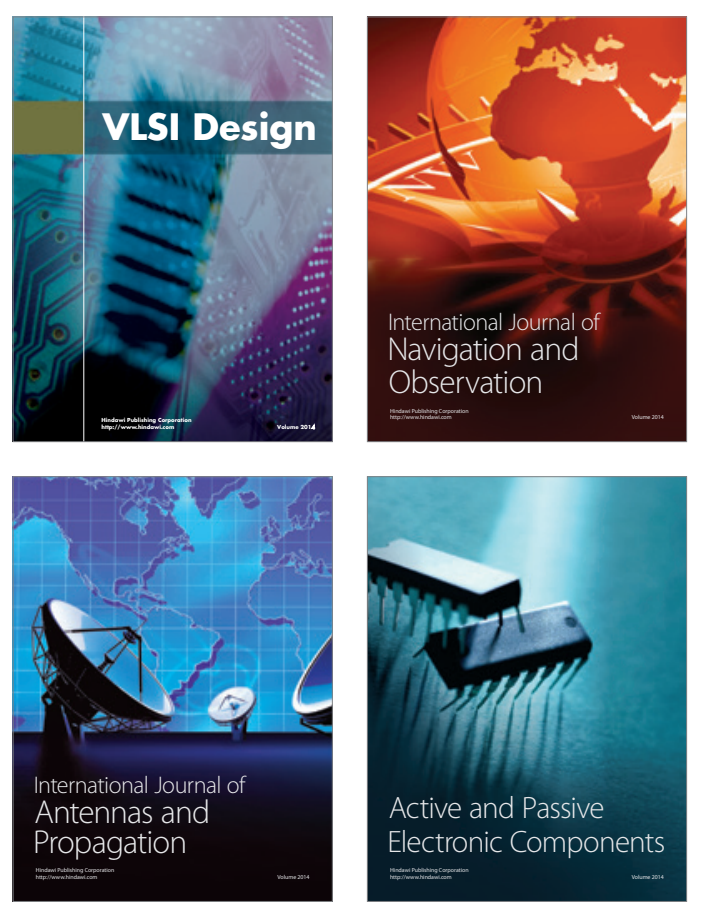
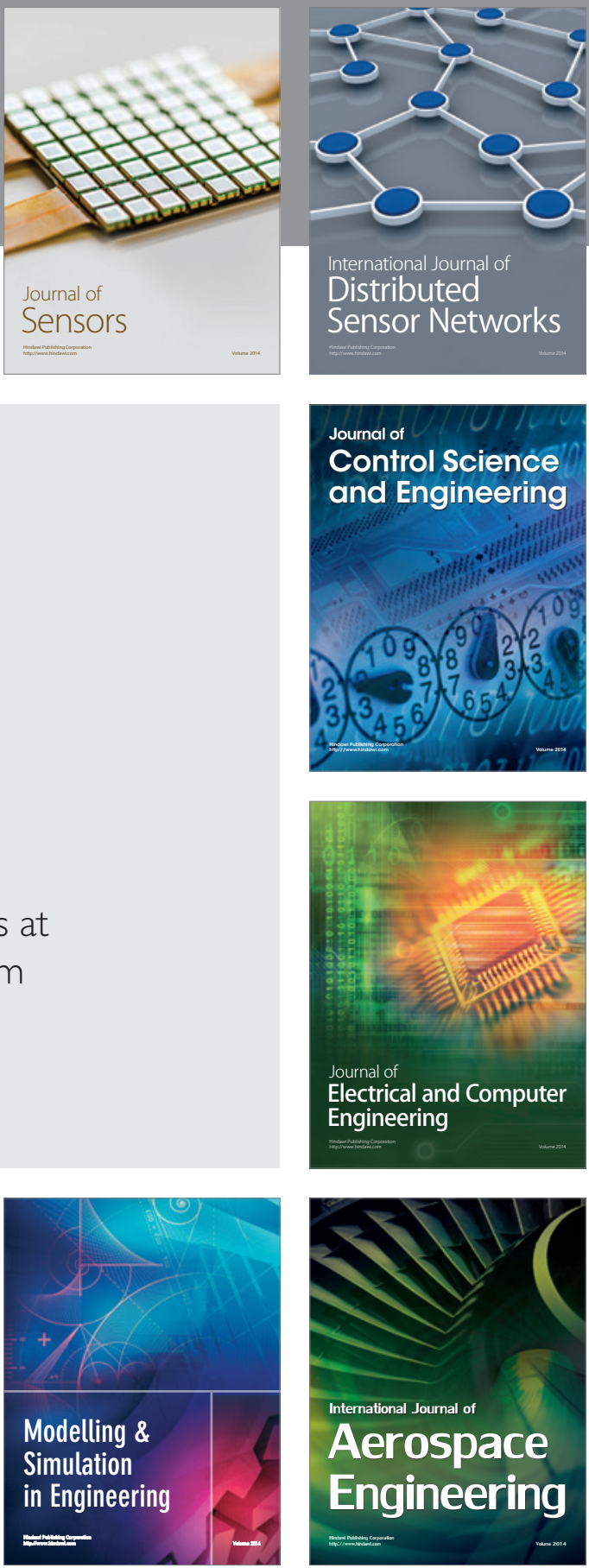

Journal of

Control Science

and Engineering
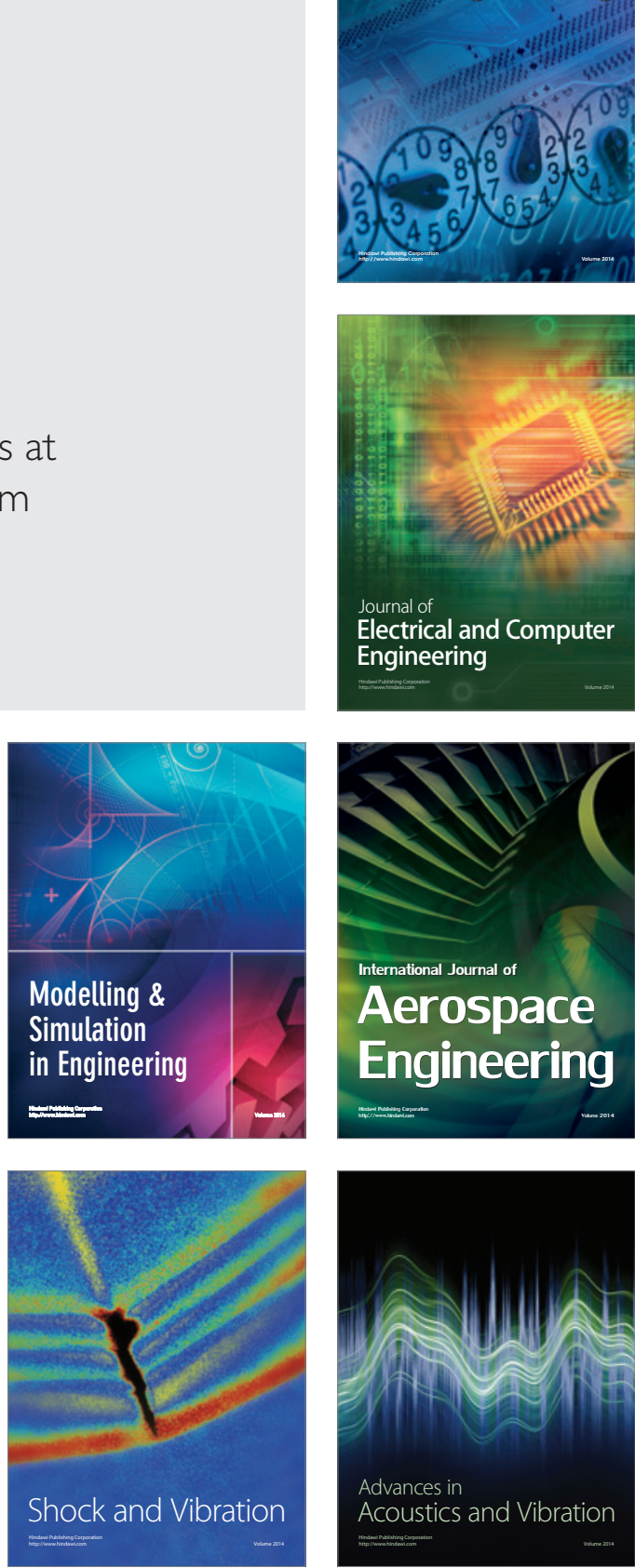